\title{
Un chantier de restauration à Angers à la fin du Moyen Âge : le compte de Jean Perier
}

A restoration worksite in Angers at the end of the Middle Ages: the accounts of Jean Perrier (1478)

Isabelle Mathieu

\section{(2) OpenEdition}

Journals

Édition électronique

URL : https://journals.openedition.org/abpo/2953

DOI : 10.4000/abpo.2953

ISBN : 978-2-7535-4130-6

ISSN : 2108-6443

Éditeur

Presses universitaires de Rennes

Édition imprimée

Date de publication : 15 avril 2015

Pagination : 41-75

ISBN : 978-2-7535-4128-3

ISSN : 0399-0826

Référence électronique

Isabelle Mathieu, «Un chantier de restauration à Angers à la fin du Moyen Âge : le compte de Jean Perier », Annales de Bretagne et des Pays de l'Ouest [En ligne], 122-1 | 2015, mis en ligne le 15 avril 2017, consulté le 28 juin 2022. URL : http://journals.openedition.org/abpo/2953 ; DOI : https://doi.org/ $10.4000 / a b p o .2953$ 


\title{
Un chantier de restauration à Angers à la fin du Moyen Âge : le compte de Jean Perier
}

\author{
Isabelle MATHIEU \\ maîtresse de conférences en histoire médiévale, Université d'Angers, \\ CERHIO UMR 6258
}

Le " Compte de Jehan Perier ${ }^{1}$ " est un registre papier de cent folios répertoriant les dépenses qui ont été engagées entre le 27 mars et le 28 septembre 1478 pour " la reparacion des foussez " situés à proximité de quatre portes (Saint-Aubin, Saint-Nicolas, Saint-Michel et Lyonnaise) de l'enceinte de la ville d'Angers ${ }^{2}$. À cette époque, l'enceinte s'étend sur 3,8 kilomètres et ne compte pas moins de sept portes principales (dont cinq en rive gauche) et sans doute quarante-cinq tours (24 sur la rive gauche et 21 sur la rive droite de la Maine) ${ }^{3}$. Protégé par une couverture en parchemin, le document est en assez bon état de conservation ${ }^{4}$.

La défense et la protection de l'enceinte d'Angers font l'objet d'une attention particulière durant tout le bas Moyen Âge. En 1473, par exemple, sur ordre du conseiller du roi, Jean des Plantes, une enquête est diligentée par un collège d'experts afin de faire le point sur l'état des murs de la cité ${ }^{5}$. C'est ainsi que,

1. Bibliothèque municipale d'Angers, ms 1148 (946). Toutes les dates sont mentionnées en nouveau style.

2. Je remercie mes relecteurs (François Comte, Isabelle Berson et Emmanuel Litoux) pour leurs remarques et leurs ajouts particulièrement pertinents.

3. Voir Сомте, François, "L'enceinte d'Angers $\left(\mathrm{XIII}^{\mathrm{e}} \mathbf{s}\right.$.) et son impact sur l'espace urbain ", dans PEDUTO, Paolo, SANTORO, Alfredo (dir.), Archeologia dei castelli nell'Europa angioina (secoli XII-XV), Florence, All'Insegna del Giglio, 2011, p. 77-89 et du même auteur "L'enceinte de Saint Louis : reconstitution d'une forteresse disparue ", dans VACQUET, Étienne (dir.), Saint Louis, roi de France en Anjou, Rennes, PUR, 2014, p. 82-92.

4. Les dimensions du document avoisinent celles d'un format légèrement supérieur à un A4.

5. Arch. dép. de Maine-et-Loire, G 400, $\mathrm{f}^{\circ} 1 \mathrm{f}^{\circ} 1 \mathrm{v}^{\circ}$; enquête publiée en annexe dans ComTE, François, "L'enceinte gallo-romaine d'Angers devenue clôture canoniale : transformations, adaptations et déclassement d'une fortification (XIII ${ }^{\mathrm{e}} \mathrm{xVI}{ }^{\mathrm{e}}$ siècles) ", dans In Situ, Revue des patrimoines, $n^{\circ} 16,2011$. Sur les enceintes urbaines en général, voir BLIECK, 
« Pierre Lemouz, macon juré, Guillaume Robin macon et savant oudit mestier, Perrinet Leverrier charpentier juré et Richart Morillon charpentier et savant oudit mestier, Jehan Bouget couvreur d'ardoise juré et Macé Regnaudier aussi couvreur d'ardoise et savant oudit mestier en la ville [sont] mandé[s pour] veoir et visiter toutes et chacunes les maisons situées et assises contre et alentour des murs de la cité d'Angiers avecques iceulx murs pour congnoistre et savoir si aucune chose auroit esté faicte ou preiudice d'iceulx et qui fust cause de la ruyne et demolicion desdits murs pour les faire reparer et remectre en estat et les choses ainsi facites à l'encontre et ou preiudice et dommaige desdits murs et saucunes en y avoit estre demolies et condemnées selon la raison, usaige et coustume du pais [...]".

Le corps de ville s'intéresse également aux fortifications urbaines comme l'attestent les registres de délibérations. Régulièrement, le mauvais état de l'enceinte est pointé du doigt, à l'instar de l'article suivant adopté à l'occasion d'une séance tenue le 28 avril 1484 :

"Item, et sur ce qu'il a esté rapporté que es tours qui sont entre les portaux de Saint Aulbin et Saint Michel y a de grans breches et perseures advenues par ruyne de la muraille qui est veille tellement que ung homme y porroit passer et s'en porroit ensuir. A esté ordonné que Jean Dupré connestable et Jehan Fallet prevost feront promptement faire et reparer les cannonieres desdites tours et reparer lesdites breches et perseures. Item, et pour faire la mise desdites reparacions et aussi les autres mises qui sont et seront à paier a esté ordonné que par Jehan Lepeletier receveur general des deniers communs sera prins et levé sur les fermiers de la Cloaison jucques à la somme de trante et cincq livres tournois ${ }^{6}$."

Le terme de Cloison renvoie au nom que l'on donne à l'enceinte mise en place par Louis IX ainsi qu'à l'impôt qui, créé en 1367 grâce à l'autorisation donnée par Charles V, a été levé afin de l'entretenir et de la restaurer. À cet égard, "Angers est un cas exceptionnel puisque rares sont les villes qui ont établi des registres de comptabilité pour leurs fortifications à part des registres communs aux autres postes du budget urbain ${ }^{7}$ ". Le principe de

Gilles, CONTAmine, Philippe, FAUCHERRE, Nicolas et MESQUI Jean (dir.), Les enceintes urbaines (XIII ${ }^{e}$ XVI $I^{e}$ siècles), Paris, CTHS, 1999. Sur l'enceinte urbaine d'Angers en particulier, voir Enguehard, Henri et Mallet, Jacques, "L'enceinte gallo-romaine d'Angers ", Annales de Bretagne, tome 71, n 1, 1964, p. 85-100; MALLET, Jacques, " Les enceintes médiévales d'Angers ", Annales de Bretagne, tome 72, n² 2, 1965, p. 237-262; ComTE, François, "L'enceinte gallo-romaine... ", op. cit., et " La défense de la Maine à Angers (XIII ${ }^{\mathrm{e}}-\mathrm{XV}^{\mathrm{e}} \mathrm{s}$.) ", dans BoIs, Jean-Pierre (dir.), La Loire, la guerre, les hommes. Histoire géopolitique et militaire d'un fleuve, Rennes, PUR, 2013, p. 75-96.

6. Arch. mun. d'Angers, BB 2, $\mathrm{f}^{\circ} 7 \mathrm{v}^{\circ}$. Une belle série de registres de délibérations est conservée. Pour un Moyen Âge long, consulter les registres BB 1 (1479-1481) à BB 23 (1544-1546). François Comte rappelle, par exemple, qu'en 1412, les réparations de quatre brèches situées entre les portes Saint-Aubin et Saint-Michel ont englouti 40 \% des sommes consacrées cette année-là aux fortifications ("L'enceinte d'Angers [XIII ${ }^{\mathrm{e}} \mathrm{s}$.] et son impact..." ", op. cit., p. 78).

7. Rousseau, Aurélien, La troisième enceinte d'Angers d'après les comptes de la Cloison (1367-1447), mémoire de maîtrise d'histoire médiévale, université d'Angers, 2002, p. 1. Ce travail a été réalisé à partir des registres de la Cloison conservés dans les archives municipales d'Angers. L'auteur rappelle que « dans le contexte très particulier de la guerre 
cet impôt est simple puisqu'il s'agit d'autoriser la ville à lever une taxe sur les marchandises entrant dans la ville ou en sortant ainsi qu'en d'autres lieux appelés quintes.

On notera d'ailleurs que les comptes de la Cloison font aussi état au $1^{\text {er }}$ août 1478 de mises " pour la reparacion des fossez et des portaux de ladite ville ${ }^{8}$ ". Certains individus cités dans ce document se retrouvent d'ailleurs dans le compte de Jean Perier : c'est le cas de Gervaise Lecamus, présenté dans les comptes de la Cloison comme " receveur des deniers communs d'Angiers pour troys ans commenczant au premier jour d'aoust M CCCC LXXV et finissant au dernier jour de juillet LXXVIII " , de " Jehan Restineau, perrier ", de " Guillaume Guerineau, charpentier ", de " Pierre Thevin et Raoullet Audouyn, commissaires " ou bien encore de "Bastien Vayre demourant à l'enseigne de l'ymaige monseigneur Saint Maurice près le portail Sainct Aubin ${ }^{10}$ ".

Les comptabilités retraçant la vie d'un chantier ne sont pas des documents singuliers : à titre d'exemple, - mais la liste pourrait être très longue -, on connaît pour le XIV ${ }^{\mathrm{e}}$ siècle, les importants comptes de construction de Macé Darne ${ }^{11}$ mais également ceux de la porte Saint-Pierre à Nantes $\mathrm{au} \mathrm{XV} \mathrm{e}^{\mathrm{e}}$ siècle $^{12}$. Outre la lecture très technique et financière que ce type de documentation invite à adopter (matériaux et outillage utilisés, techniques

de Cent Ans et des défaites françaises qui émaillent le début du conflit (batailles de l'Écluse 1340, de Crécy 1346, de Calais 1347 et de Poitiers 1356), le 14 mai 1358, par une ordonnance, toutes les forteresses passent sous contrôle royal : le roi peut désormais décider de leur restauration ou de leur destruction. Une seconde ordonnance du 19 juillet 1367 donne l'ordre à toutes les bonnes villes d'assurer elles-mêmes l'entretien de leurs fortifications".

8. Arch. mun. d'Angers, CC 5, $\mathrm{f}^{\circ} 32$.

9. Arch. mun. d'Angers, CC $5, \mathrm{f}^{\circ} 32$.

10. Arch. mun. d'Angers, CC 5, $\mathrm{f}^{\circ} 45-46$.

11. Pour le XIV ${ }^{\mathrm{e}}$ siècle, nous disposons, par exemple, des comptes de Macé Darne, maître des requêtes de Louis I $^{\text {er }}$ d'Anjou. Ils sont conservés à la British Library, sous la cote add. Ms. 21201 (microfilm disponible aux Arch. dép. de Maine-et-Loire, 1Mi 37). Jacques Mallet a réalisé la transcription intégrale des articles relatifs à des dépenses engagées sur plusieurs chantiers ducaux en Anjou-Maine (f 82 à 160), voir Arch. dép. de Maine-et-Loire, BIB 10717, Les comptes de Macé Darne, Angers, 2000, dactyl.; JOUBERT, André, Étude sur les comptes de Macé Darne, maître des œuvres de Louis I ${ }^{\text {er }}$, duc d'Anjou et comte du Maine (1367-1376), d'après un manuscrit inédit du British Museum, Angers, 1890. On pourrait également citer le travail sur le château de Beaufort-en-Vallée mené, entre autres, à partir de comptes de construction (GuITON, Arnaud, La reconstruction du château de Beauforten-Vallée d'après un livre de comptes de 1348, mémoire de maîtrise, université de Tours, 1988) ou ceux de Robin, Françoise, "Les chantiers des princes angevins (1370-1480): direction, maitrise, main-d'œuvre ", Bulletin Monumental, tome 141, 1983, p. 21-65 et de Litoux, Emmanuel, CRon, É. (dir.), Le château et la citadelle de Saumur. Architectures du pouvoir (supplément au Bulletin Monumental), Paris, Société française d'archéologie, 2010.

12. ARmide, Aurélien, La porte Saint-Pierre à Nantes : étude architecturale d'un édifice militaire fortifié urbain du Xve siècle en Bretagne, mémoire de maîtrise d'archéologie médiévale, 2 vol., université Paris Iv Sorbonne, 2005. Voir également SALAMAGNe, Alain, Construire au Moyen Âge. Les chantiers de fortification de Douai, Paris, Presses universitaires du Septentrion, 2001. 
de construction mises en œuvre, coût de la main d'œuvre et des travaux effectués...), il est possible d'en faire une analyse davantage sociale, tournée vers les individus concernés, à un titre ou à un autre, par ces travaux.

Formellement, le registre se présente de manière très structurée. Il est organisé autour de la succession de paragraphes matérialisés par des espaces blancs et parfois l'utilisation du mot item. Les notes marginales montrent que l'on se trouve en présence d'un document de la pratique qui a été à plusieurs reprises consulté et retouché. Le document s'ouvre par les lettres de commission de Jean Perier, sorte de procès-verbal d'installation dans ses fonctions de " commis pour faire la mise de la reparacion des foussez d'Angiers ${ }^{13}$ ". Ensuite, ce sont plusieurs marchés passés entre la ville et différents corps d'artisans qui ont été reportés, puis viennent les comptes à proprement parler, soit les recettes, très courtes dans l'exposé (environ un folio) et les dépenses qui ont été affectées à la réparation de la muraille et des fossés d'Angers. À droite, en bout de ligne, sont indiquées les sommes dépensées. Des totaux intermédiaires sont régulièrement faits, ce qui permet de suivre pas à pas les dépenses. En fin de registre, figure la somme globale des recettes et des coûts des travaux.

Avant d'entrer dans le détail de ce document, il convient de s'interroger sur ce qui a pu motiver le lancement de ces réparations. En 1478, cela fait 25 ans que la guerre de Cent Ans est terminée. L'Anjou n'a pas été continuellement affecté par les conflits qui s'étalent de 1337 à 1453. Malgré des heures difficiles à certains moments, une phase intense de réparations et de reconstructions en tout genre a été amorcée assez rapidement. Pour autant, les escarmouches militaires n'ont pas totalement déserté la province. En effet, comme l'écrit très justement Michel Le Mené, " du début de la guerre du Bien Public au règlement de l'affaire bretonne ${ }^{14}$ sous Charles VIII, l'Anjou connaît une longue période d'insécurité. En 27 ans, de 1465 à 1491, il n'y eut pas moins d'une bonne dizaine d'années marquées, d'une part, par le passage et le stationnement d'importantes troupes dans le duché et, d'autre part, par les courses dévastatrices des ennemis et des pillards de tout genre. [...] Tout déplacement de gens d'armes réveillait la crainte et les inquiétudes souvent justifiées des urbains et des villageois. [...] C'est principalement à la tension des relations franco-bretonnes, qui ne cessè-

13. Cette information est visible directement sur la couverture même du registre.

14. L'affaire bretonne débute avec le décès de François II en septembre 1488, des suites d'une chute de cheval. Sa fille, Anne, devient de fait duchesse de Bretagne; elle est placée sous la tutelle du maréchal de Rieu. La Bretagne devient alors le théâtre de toutes les convoitises. Le roi, Charles VIII, demande à plusieurs généalogistes de travailler afin de faire valoir ses droits sur le duché et de nombreux princes européens convoitent également la main de la jeune duchesse. On peut même dire qu'il y a du monde sur les rangs : Angleterre, Espagne, Autriche. En décembre 1491, Charles VIII parvient à épouser Anne, faisant entrer de fait la Bretagne dans le domaine royal. Les clauses du mariage prévoyaient que si Anne mourait la première, le duché deviendrait la propriété de Charles VIII mais que si le roi décédait avant son épouse sans enfant, Anne devrait épouser le nouveau souverain. 
rent de se détériorer à partir de 1462, que la province dut de connaître une nouvelle phase de troubles ». En effet, Louis XI s'est lancé dans une délicate opération contre le duché de Bretagne : celle de réduire ses velléités d'indépendance. Son objectif consiste à rattacher la province au domaine royal, dessein qui a eu pour conséquences de voir éclater plusieurs phases de conflits armés, qui ne se sont véritablement achevés qu'avec le mariage de Charles VIII et d'Anne de Bretagne en 1491.

Dans cette affaire bretonne, l'Anjou est concerné pour deux raisons : la première est géostratégique puisque la province est un passage quasiment obligé sur le chemin de la Bretagne; la seconde tient au fait que le duc d'Anjou, René ${ }^{15}$, est l'oncle du roi et à ce titre le souverain compte sur son soutien. D'ailleurs, Louis XI regarde aussi du côté de l'Anjou. Soucieux de réunir cette province à la couronne royale, Louis XI a habilement manœuvré, et de plus en plus ouvertement, contre son vieil oncle. Prétextant des dispositions testamentaires prises par René qui lui étaient défavorables, il fait tout d'abord saisir le duché en juillet 1474; il nomme immédiatement un nouveau gouverneur - Guillaume Cerisay - et envoie un lieutenant général. L'année suivante, en février, il institue la municipalité, ce qui contribue à renforcer son emprise sur l'Anjou. Retiré depuis 1471 en Provence, René meurt quelques années plus tard, ce qui marque l'ultime étape du rattachement de l'Anjou au domaine royal (1481).

L'apparition en 1474-1475 d'un " commis au paiement de l'avitaillement et des fortifications du château d'Angers " laisse penser que des travaux importants sont engagés ou, à tout le moins, envisagés dans ces mêmes années. Le contexte politique tendu avec la Bretagne incite Louis XI à faire d'Angers une base arrière pour ses campagnes militaires, et à mettre à profit le potentiel défensif du château pour y établir un arsenal. En 1477, il interdit que les recettes de la Cloison d'Angers soient employées à autre chose que l'ouvrage des fossés qui font l'objet de travaux importants en $1478^{16}$. Le compte de Jean Perier s'inscrit très clairement dans ce contexte particuliers de tensions qui se maintiennent d'ailleurs encore pendant quelques années. En effet, Lecoy de la Marche rappelle, par exemple, que, le 28 octobre 1481, Louis XI ordonne que " la somme de deux cent livres soit prise chaque année sur les droits de ventes et rachats perçus en Anjou pour être employée aux réparations du château d'Angers ${ }^{17}$ ". On sait par ailleurs que d'autres travaux sont menés, par exemple sur le ravelin de la porte des champs ${ }^{18}$.

15. Fils de Louis II et de Yolande d'Aragon. Charles VII est marié avec sa sœur Marie. Naît de ce mariage Louis XI (1461-1483).

16. LE MENÉ Michel, Les campagnes angevines..., op. cit., p. 257, note 31.

17. LECOY DE LA MARCHE, Albert, Extraits des comptes et mémoriaux du Roi René pour servir à l'histoire des arts au XV viècle, Paris, Alphonse Picard, 1873, p. 21-22.

18. Sur les progrès de la fortification à cette période, voir par exemple FAUCHERRE, Nicolas, Les citadelles du roi de France sous Charles VII et Louis XI, thèse de doctorat d'archéologie médiévale, université de Paris I Panthéon-Sorbonne, 1993, 3 vol. 
On le voit, le contexte en Anjou à la fin du Xve siècle est suffisamment menaçant pour justifier des réparations des fossés et des murs de la muraille ainsi que des aménagements au château. Ces quelques éléments de contexte posés, nous nous proposons de faire une analyse en quatre temps de ce document comptable. Il s'agira de montrer que ces travaux sont entrepris dans le cadre d'une procédure strictement définie et conduits sous l'égide d'individus choisis à cet effet (I) mais aussi de rendre compte tant de la nature des travaux qui ont été engagés (II) que des moyens matériels et humains qui ont été mobilisés (III); enfin, ce document donne accès à des aspects de la vie quotidienne d'un chantier à la fin du XV siècle (IV).

\section{La mise en place des travaux : une procédure bien conçue}

La consignation par écrit de ces travaux appelle quelques remarques. La lecture attentive du registre laisse penser que les individus impliqués dans ce chantier, qu'il s'agisse des commanditaires ou des exécutants, ont conscience qu'un cahier des charges précis est la garantie que les travaux se dérouleront bien. Les notes ajoutées en marge prouvent toutefois que tout n'a pas été prévu dans les moindres détails dans le document original. Ainsi, à plusieurs reprises, il a fallu, par exemple, préciser certains aspects du chantier comme la restitution de certains matériels ou les lieux qui ont été choisis pour servir d'entrepôts. L'intérêt d'un tel document réside également dans le fait qu'il rend aisé le suivi des sorties d'argent, qu'il s'agisse de payer les matériaux ou de verser les salaires des ouvriers. L'objectif assigné à ce registre semble clair : il s'agit de permettre de mener les travaux dans les meilleures conditions et les délais les plus justes et de rendre possible le contrôle de l'utilisation qui est faite de l'argent dévolu à ces réparations; une mission qui est confiée à Jean Perier. Le registre le présente comme étant un " marchant demourant en ceste dicte ville d'Angers ${ }^{19}$ ". Commissaire, il tient sa mission des " maire, eschevins et conseillers de la ville d'Angiers ${ }^{20}$ " dont les noms sont explicitement reportés dans le compte. Sont également mentionnées les personnes chargées de se rendre sur place pour vérifier les travaux ainsi que la présence de greffiers et d'un notaire, ce dont rend compte le tableau page suivante.

Ce microcosme d'une trentaine de personnes au total est donc là aux côtés de Jean Perier pour prendre en charge, à des degrés d'implication divers, les réparations. Les plus hautes autorités de la ville, le maire et les échevins notamment, sont bien parties prenantes de l'opération, ce qui atteste du caractère très encadré de l'entreprise ${ }^{21}$. La sécurité de la ville, autant celle de sa population que celle de ses infrastructures, est un

19. Bib. mun. d'Angers, ms 1148 (946), $\mathrm{f}^{\circ} 1$.

20. Ibidem, $\mathrm{f}^{\circ} 1$.

21. Célestin Port a rédigé des notices biographiques pour plusieurs d'entre eux (Guillaume Cerisay, Bertrand Duvau, Jean Lohéac...) (PorT, Célestin Dictionnaire historique, géographique et biographique de Maine-et-Loire, Paris/Angers, 1869-1878, 3 vol.; nouvelle édition, Angers, H. Siraudeau, 1965-2004, 6 vol.). Voir également BERSON, Isabelle, 
Un chantier de restauration à Angers à la fin du Moyen Âge

\begin{tabular}{|c|c|}
\hline Prénom/Nom & Qualité mentionnée dans le registre/Fonction \\
\hline Guillaume Cerisay & Conseiller du roi, maire \\
\hline Jean Barrault & $\begin{array}{l}\text { Bourgeois de l'Université } \\
\text { Les bourgeois de l'Université sont également appelés suppôts. } \\
\text { échevin, en charge de la garde des objets au portail Saint- } \\
\text { Aubin }\end{array}$ \\
\hline Jean Bernart & Élu d'Angers et commissaire \\
\hline Pierre de Chartres & $\begin{array}{l}\text { Connétable } \\
\text { À cette époque, la fonction recouvre des fonctions militaires } \\
\text { souvent attachées à un secteur de la ville localisé à proximité } \\
\text { d'une porte (connétable de la porte Lyonnaise, de la porte } \\
\text { Saint-Michel, de la porte Saint-Aubin...). } \\
\text { Échevin }\end{array}$ \\
\hline Jean du Chasteau & Maître, licencié en lois \\
\hline Jean Delaunay & Échevin, conseiller et député par le conseil de ville \\
\hline Bertrand Duvau & Maître, élu d'Angers et commissaire \\
\hline Jean Ernault & Échevin, conseiller et député par le conseil de ville \\
\hline Jean Fallet & Maître, échevin et conseiller, prévôt \\
\hline Pierre Guiot & Maître, échevin et conseiller \\
\hline Gervais Lecamus & Bourgeois, échevin \\
\hline Guillaume Leroy & Échevin \\
\hline Jean Lohéac & $\begin{array}{l}\text { Maître, échevin et conseiller du roi et garde de la prévôté d'An- } \\
\text { gers }\end{array}$ \\
\hline Jean Muret & Maître, échevin et conseiller \\
\hline François Prévost & Sire de Bonnezeaux, connétable et échevin \\
\hline Jean de la Rivière & Échevin, conseiller et député par le conseil de ville \\
\hline René Touscherousche & Échevin, conseiller et député par le conseil de ville \\
\hline Bastien de Losche & Connétable \\
\hline Jean Ferrault & Garde la monnaie \\
\hline Jean Rambaut & Receveur de la mairie \\
\hline Thibault Lemaczon & Maître, licencié en lois, procureur \\
\hline Hilaire Cadu & Maître, greffier \\
\hline Michel Gillebert & Greffier \\
\hline Jean Marsault & Notaire \\
\hline Pierre Thevin & \multirow{6}{*}{$\begin{array}{l}\text { Commissaires et députés, chargés de vérifier les travaux avec/ } \\
\text { pour Jean Perier }\end{array}$} \\
\hline Raoulet Audoyn & \\
\hline Jean Henry & \\
\hline Jean Lecommandeurs & \\
\hline Jean Nicholas & \\
\hline Étienne Fleury & \\
\hline
\end{tabular}

Les élites municipales d'Angers de 1475 à 1540, mémoire de master 2, université d'Angers, 2012. 
sujet sérieux qui mobilise l'attention de toute une frange de la notabilité angevine.

Le compte est précis à propos de la mission qui incombe à Jean Perier : si ce dernier ne semble pas prendre part aux choix techniques ni aux négociations ayant trait aux marchés, en revanche, le corps de ville attend de lui qu'il suive minutieusement les travaux. Il a été " commis pour faire les paiemens de toute la despence qui sera faicte pour la reparacion des fossez de ceste dicte ville d'Angiers, dujourd'uy jucques à la Toussains prouchain venant sur les deniers communs de ladite ville ${ }^{22}$ ". Ses obligations consistent à dresser des " quictance aux parties à qui il fera les paiemens signée ", des quittances dont on précise qu'elles devront être signées de la main du " clerc et greffier de la ville ou d'autre notaire en son absence et des commissaires ordennez pour veoir la mise et despence desdits fos$\operatorname{sez}^{23}$ ». Dans les lettres de commission de Jean Perier, il est précisé qu'une somme de " mil cinq cens quatre vigns quinze livres tournois, trois soulz, neuff deniers ${ }^{24}$ " lui sera " baillez et avancez " par Gervaise Lecamus et le maire d'Angers, à laquelle s'ajoutent 46 livres versées par Jean de la Rivière échevin, à charge pour lui de "fere lesdites mises et despence ".

Au début du registre, en marge, à gauche des lettres de commission de Jean Perier établies à Angers le " 27 mars 1478 après Pasques ", on peut d'ailleurs lire cette note amusante, reportée par le greffier en charge de la tenue de ce registre : "Veu la commission dudit Perier. Il commance bien ", tandis que celle de droite permet de constater que ce compte a été " presenté par ledit Jehan Perier en l'ostel de la ville le 17 mars 1484 es presences de honnorables hommes et sages maistres Pierre Guiot, Jehan Loheac, Jehan Muret et Jehan Fallet eschevins et conseillers de ladite maerie ". Le compte a visiblement fait l'objet d'une présentation devant le corps de ville, ce qui est une nouvelle fois mentionné au dernier folio du registre puisque l'on peut lire que : « oyz, cloz et examinez ces presens comptes en la maison de la mairie d'Angiers par nous esleuz pour le roy notre Sire en la ville et ellection dudit lieu d'Angiers et commissaires par luy ordonné en ceste partie es presences de [...], le XIII jour de novembre l'an cccc quatre vings et cincq ${ }^{25}$ ". Comme il se doit, le tout est accompagné de plusieurs signatures : celles de Béruart et Duvau, deux échevins, et de H. Cadu le greffier.

Tout au long du registre, il ressort que la municipalité a imposé un certain nombre de vérifications, comme s'assurer du respect de la conformité des travaux qui ont été prévus sur le papier. Ainsi, " lesdits Thenin et Fallet, les commissaires, ont certiffié à bouche à l'audicion de ce compte avoir esté presens et que les ouvriers dessus declerez ont besongné esdits

22. Inscription visible sur la couverture même du registre.

23. Bib. mun. d'Angers, ms 1148 (946), $\mathrm{f}^{\circ} 1$.

24. Ibidem, $\mathrm{f}^{\circ}$ 2, marge de gauche, « veu le compte de Jehan Rambaut receveur de ladite maerie, la somme contenue en cest article est vraye".

25. Ibidem, $\mathrm{f}^{\circ} 100$. 
foussez par les journées dessusdites ${ }^{26}$ ». De même, ils ont affirmé que " chacun article contient verité et les marchez en iceulx avoir esté faiz aux sommes de deniers dedens contenu et les ouvrage faiz et acompliz, ferreures, cyvieres et autres choses pour ce achapter avoir esté baillées et livrées et d'icelles fait les pris et marchez aux sommes cy après employées en despence en chacun desdits articles ${ }^{27}$ ".

Les réparations se font dans le cadre de marchés contractés entre deux parties : la mairie d'Angers et divers artisans. Des marchés sont ainsi passés avec deux perriers ${ }^{28}$ (" Jean Restineau demeurant en la parroisse SaintNicolas et Alain Audouart demeurant en la parroisse de Saint-Michel-duTertre "), quatre maçons (Thibaut Belin et Étienne, son fils, de Laval ${ }^{29}$, Jean Roger et Jean Lore de Soucelles ${ }^{30}$ ) et trois terrassiers (Yvonnet Lemoulnier, Jean Lejars et Noël Richart). Ces contrats décrivent le type de travaux à faire, les matériaux à utiliser, le temps à passer, le nombre d'hommes nécessaires ainsi que le coût, sachant que chaque marché fait ensuite l'objet de vérifications et éventuellement d'ajustements financiers ${ }^{31}$.

Ce registre n'est pas l'unique document papier produit dans le cadre de la mise en œuvre de ces réparations. En effet, à plusieurs reprises, le greffier précise par exemple que " le marché est escript en ung cayer de pappier ouquel sont autres marchez escriptz et signés dudit Gilbert servans cy après pour les parties de Alain Audouart, Thebaut Belin, Jehan Rogier et Jean Lori contenant en oultre paiemens faiz par iceluy Perier à plusieurs macons et bessons ${ }^{32}$ ". Malheureusement, ni ces cahiers, ni les quittances de paiement n'ont été retrouvés. Dans le même ordre d'idées, à la fin du compte, on peut lire que ce compte a été fait en plusieurs exemplaires (un original et plusieurs copies) $)^{33}$.

Sans nul doute, et à rebours de ce que l'on entend encore trop souvent, l'écrit tient une place importante dans la société médiévale. Très tôt, les hommes et les femmes ont bien compris l'intérêt qu'il y avait de consigner des informations jugées par eux importantes. Ainsi manipulent-ils à l'envie les contrats sous toutes leurs formes : ventes, mariages, métiers, travaux, marchés, rentes etc. L'écrit permet de formaliser les paroles échangées. Garder une trace écrite authentifiée par des signes de validation, comme les signatures, permet, le cas échéant, de remettre en cause des sommes indûment perçues, d'attester les salaires reçus, de contester la qualité des matériaux employés, voire d'exiger de refaire des travaux qui auraient été

26. Ibid., $\mathrm{f}^{\circ} 30$.

27. Ibid., $\mathrm{f}^{\circ} 30 \mathrm{v}^{\circ}$.

28. Un perrier est un carrier.

29. LAVAL, Mayenne, ch.-l. de dép.

30. SouCELLES, Maine-et-Loire, arr. Angers, cant. Tiercé.

31. On peut lire par exemple dans la marge du $\mathrm{f}^{\circ} 10 \mathrm{v}^{\circ}$ que « Perier est chargé informer par certifficacion comme dessus que ledit Audouart ait acomply sondit marchié ".

32. Bib. mun. d'Angers, ms 1148 (946), f ${ }^{\circ}$. Un besson est un terrassier.

33. Ibid., $\mathrm{f}^{\circ} 100$. 
éventuellement mal faits. Ceci montre clairement que, dans l'esprit des médiévaux, l'écrit protège.

\section{La nature des travaux engagés}

Même si, à cette époque, beaucoup de villes mettent en œuvre ce genre de défenses, le chantier angevin impressionne par son ampleur : cela se voit tant du point de vue des corps de métiers mobilisés et du nombre d'individus recrutés que des travaux entrepris, des matériaux nécessaires et de l'argent dépensé.

\section{Des chantiers multiples, une organisation complexe}

Les travaux ne sont pas concentrés à un endroit précis de la muraille. Bien au contraire, ils sont répartis, comme l'atteste le plan suivant, en différents lieux de l'enceinte, à savoir à proximité des portes Saint-Aubin, SaintMichel, Saint-Nicolas et Lionnaise, - des portes qui se trouvent à l'opposé les unes des autres -, ce qui complique l'organisation du chantier. Ainsi, en termes logistiques, cela signifie que des matériaux ont dû être acheminés à plusieurs endroits, et qu'il a fallu en outre évaluer les volumes spécifiques en fonction des tâches à réaliser.

Les marchés permettent de situer de manière très précise les travaux. Par exemple, un entrepreneur s'est engagé à " perroier ou fossé du portal Saint Aulbin depuis le bort de la fosse qui est près le grant monteau de pierre jucques au bout de l'emparement (contrescarpe maçonnée) qui a esté fait au droit de l'eglise de monseigneur Saint Sauveur qui contient en longueur dix et sept toises ou environ chacune toise de sept piez et demy de long et le parfondera aussi bas que Restineau doit faire sa tasche et de la largeur à la raison de ladite tasche dudit Restineau ${ }^{34}$ ", tandis que plusieurs maçons ont travaillé à " faire de la main seulement ung pan de muraille près le petit boullevart du portal Saint Aubin commanczant au mur que fist faire le feu commissaire et finissant à ung pal de boais fourche qui est frappé ou mur à sec et contient ledit pan en longueur quatorze toises ou environ à VI piez pour toise et aura quatre piez de largeur sur le nayff et en amortissant jucques à la haulteur de l'autre mur en maniere qu'il ayt trois piez de large par le hault et lyeront ledit pan de mur avecques l'autre du bout dudit boulevart ${ }^{35}$ ". Plus loin dans le registre, on apprend que des dépenses ont également été faites pour la «muraille [qui est] entre le portal Saint Nicholas et le portal Leonnays ${ }^{36}$ ".

Enfin, l'indication reportée sur la couverture du registre "Compte de Jehan Perier commis pour faire la mise de la reparacion des foussez d'Angiers pour l'année mil IIII ${ }^{c}$ LXXVIII " ne rend pas tout à fait compte de la réalité

34. Bib. mun. d'Angers, ms 1148 (946), f ${ }^{\circ} 8$.

35. Ibid., $\mathrm{f}^{\circ} 11$.

36. Ibid., $\mathrm{f}^{\circ} 21$. 
Figure 1 - Angers à la fin du $X V^{e}$ siècle

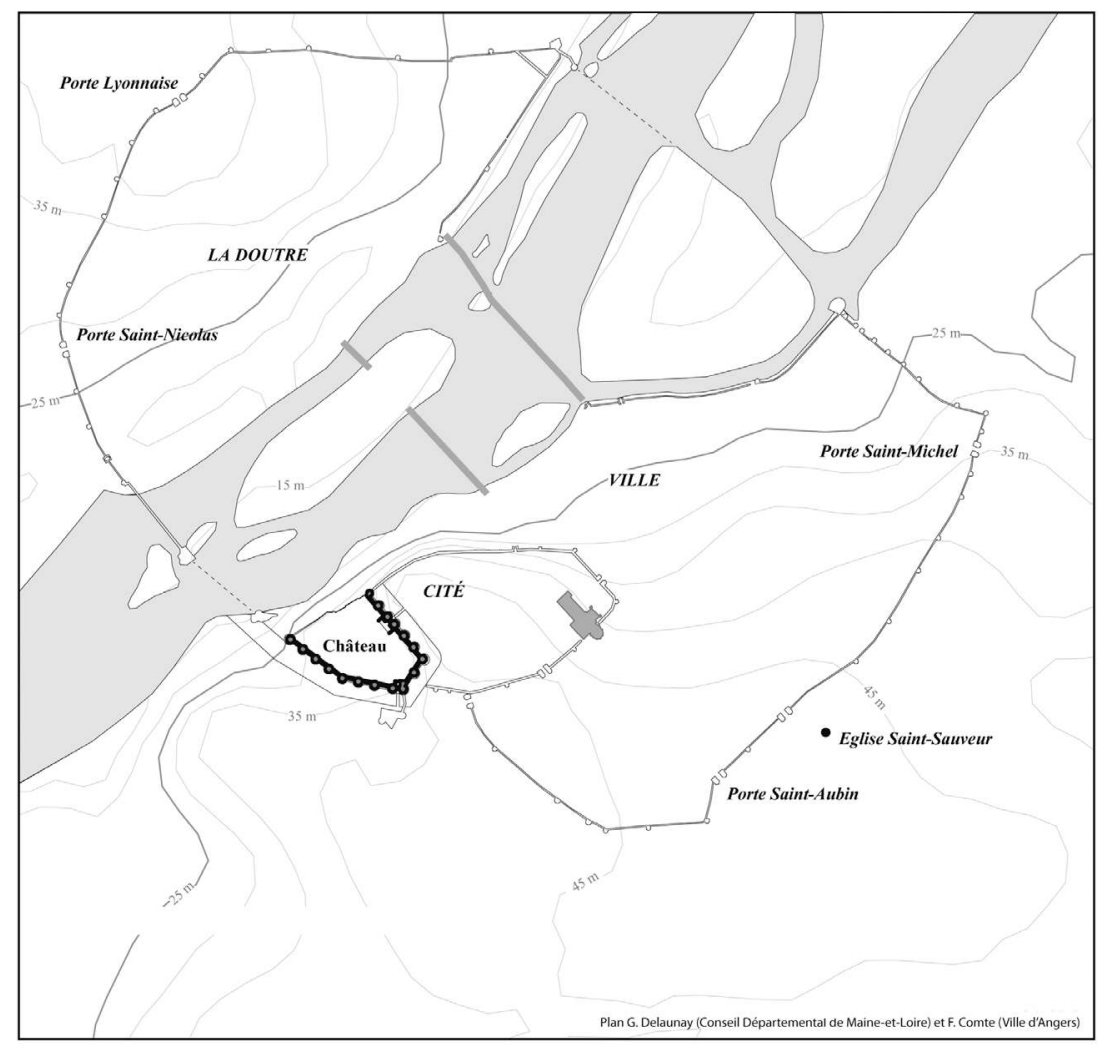

des travaux qui ont été faits. En effet, si les fossés font bien l'objet d'un certain nombre de réparations, l'enceinte, notamment ses portes, est également concernée. En revanche, le compte ne dit rien d'éventuels travaux effectués sur les tours.

\section{Les temps du chantier}

Si la comptabilité s'étale de mars à septembre, soit sur sept mois, en réalité les ouvriers travaillent d'avril à la fin de la troisième semaine de septembre, soit six mois ou un total de 25 semaines. Ce mois d'écart n'est pas expliqué : peut-être sert-il à mettre en place le budget, à collecter les fonds, à recruter les ouvriers ou bien encore à commander les matériaux.

Le temps de travail oscille entre 3 et 6 jours hebdomadaires sur le chantier, même si, le plus souvent, les ouvriers sont présents 6 jours par semaine ce qui n'est pas la norme admise à cette époque, qui est plutôt de 5 jours de travail hebdomadaire en moyenne. Comment expliquer ce temps de travail 
supérieur à la moyenne habituelle? Sans doute les ouvriers avaient-ils des délais de livraison des travaux à respecter. Peut-être étaient-ils également contraints par le climat à terminer les travaux avant l'arrivée de l'automne et des pluies qui l'accompagnent généralement.

À cette époque, une année comprend de 250 à 260 jours ouvrables et entre 100 et 110 jours de repos forcé ${ }^{37}$. Les jours chômés sont en lien avec le calendrier liturgique (dimanches, Pâques, Pentecôte, Noël, etc.) et la vie communautaire (veilles ou lendemains de réjouissances publiques, de marchés ou de foires, jour de la fête corporative, mariages, baptêmes ou enterrements, phénomènes climatiques...). En l'occurrence, sur ce chantier, les jours traditionnellement chômés ne sont pas forcément respectés. En effet, certains ouvriers travaillent le dimanche : c'est le cas d'une petite dizaine d'individus dont le registre précise explicitement qu'ils travaillent les " dimanche, lundi, mardy, mercredi, jeudi, vendredi et sabmedi ${ }^{38}$ ". Ils appartiennent à une catégorie d'individus très particulière sur laquelle nous reviendrons plus loin; pour le moment, disons seulement qu'il s'agit d'individus qui sont en compte avec la justice. D'autres ouvriers sont amenés à travailler la nuit : c'est le cas de Jehan Theon qui est payé 5 sous " pour avoir été ung jour et deux nuytz au devant de la chaux ${ }^{39}$ ", de Raoullet Regnouf et Étienne Chaptel qui reçoivent également 5 sous chacun " pour avoir esté par deux nuytz à tyrer [l'eau des fossés] ${ }^{40}$ ". Le travail la nuit est à mettre en lien avec des tâches très spécifiques nécessitant la présence jour et nuit d'individus pour éteindre la chaux ou empêcher que l'eau ne s'accumule dans les zones d'extraction au fond des fossés.

Si le temps de travail journalier n'est pas abordé dans le compte, des études ont montré qu'il est soumis à des rythmes saisonniers qui prennent en compte la nuit tombante ou bien encore les intempéries ${ }^{41}$. En moyenne, il serait de l'ordre de 9 à 12 heures en hiver, de 14 à 16 heures en été; mais ces amplitudes horaires ne constituent pas le temps réel de travail car on doit, par exemple, retrancher le temps réservé aux repas (la collation du matin, prise vers 9 ou 10 heures, le repas de midi ou de 13 heures, la pause de l'après-midi vers 15 ou 16 heures), de sorte que, ces pauses retirées, le temps de travail en été est estimé aux alentours de 12 heures et en hiver il

37. Sur la répartition entre jours ouvrables et jours chômés, voir les cas concrets analysés par GuitTon, Arnaud, La reconstruction du château de Beaufort-en-Vallée..., op. cit., p. 214-216 et GEREMEK, Bronislaw, Le salariat dans l'artisanat parisien aux XIII $-X V^{e}$ siècles. Études sur le marché de la main d'œuvre au Moyen Âge, Paris, Mouton et Co, 1968, p. 84. Voir à titre de comparaison LARDIN, Philippe, "Le temps de travail sur les chantiers du bâtiment normands à la fin du Moyen Âge ", dans MAZAuric, Claude (dir.), Temps social, temps vécu, Actes du $129^{\mathrm{e}}$ Congrès des sociétés historiques et scientifiques (Besançon, 2004), Paris, CTHS, 2007, p. 147-161.

38. Bib. mun. d'Angers, ms 1148 (946), f 79.

39. Ibid., $\mathrm{f}^{\circ} 91$.

40. Ibid., $\mathrm{f}^{\circ} 57 \mathrm{v}^{\circ}$.

41. Sur le temps de travail, à titre de comparaison, voir GEREMEK, Bronislaw, Le salariat..., op. cit., p. 78-82, et BERnARDI, Philippe, Bâtir au Moyen Âge, Paris, CNRS Éditions, rééd. 2014, p. 107-113. 
est peut-être de l'ordre de 10 heures $^{42}$, ce qui fait de longues journées de labeur.

\section{Diversité des travaux engagés}

Le compte de Jean Perier montre que les chantiers répartis aux différents endroits de l'enceinte sont bien organisés, les ouvriers ayant à accomplir des tâches clairement définies. Précisons que cette campagne de travaux qui a lieu en 1478 n'est pas une première. En effet, comme le rappelle François Comte, " les comptes de la Cloison nous apprennent qu'un siècle plus tôt, en 1377-1378, ce sont près de la moitié des fossés qui ont été vidés et recreusés, ce qui a nécessité la mise en place de corvées dans la quinte mobilisant quarante à cinquante personnes en continu pendant plusieurs semaines tandis qu'en 1412, ce sont trois cent quarante-cinq hommes qui curent les douves de la Tannerie ${ }^{43}$ ".

\section{Préparer le terrain}

Avant même de faire les travaux à proprement parler, des travailleurs sont préposés à la mise en place d'installations nécessaires à la bonne conduite des opérations. Il faut par exemple monter des échafaudages, ce qui vaut à Jean Hérault d'être rémunéré 15 sous tournois, le 2 mai, " pour deux charretées de perches pour faire les chauffault des maczons qui besongnent esdits fossez ${ }^{44}$ ". Si Guillaume Thomin a " besongné deux jours au portal Saint Michel à faire les chemins pour curez la douve soubz le premier pont dudit portau ${ }^{45}$ ", d'autres ouvriers sont chargés d'installer deux " engins ", un " petit et un grant ${ }^{46}$ ", à proximité des " douves du portal Saint Aulbin $^{47}$ ". En août 1478, Guillaume Guérineau et " ses gens " sont d'ailleurs payés pour " remuez le petit engin et [le mettre] près le grant engin ${ }^{48}$ ". Le but de telles installations est de faciliter les travaux de force consistant, par exemple, à transporter des charges lourdes, qu'il s'agisse de remonter la pierre extraite du fond des fossés ou d'acheminer des matériaux.

42. LEGUAY, Jean-Pierre, "Les ouvriers des chantiers de construction nantais au $\mathrm{XV}^{\mathrm{e}}$ siècle ", 303 Arts, recherche, création, $\mathrm{n}^{\circ}$ 92, 2006, p. 9.

43. Comte, François, "L'enceinte d'Angers et son impact... ", op. cit., p. 79, d'après Rousseau, Aurélien, La troisième enceinte d'Angers..., op. cit.

44. Bib. mun. d'Angers, ms 1148 (946), $\mathrm{f}^{\circ} 40 \mathrm{v}^{\circ}$.

45. Ibid., $\mathrm{f}^{\circ}$ 43. La mention d'une douve confirme la présence de l'eau sous le pont dormant de la porte Saint-Michel (ComTE, François, "L'enceinte de Saint Louis... ", op. cit., p. 88).

46. Bib. mun. d'Angers, ms 1148 (946), $\mathrm{f}^{\circ} 40$.

47. Ibidem, $\mathrm{f}^{\circ} 48 \mathrm{v}^{\circ}$. Sur les engins de levage utilisés sur les chantiers médiévaux, voir Bernard, Philippe, Bâtir au Moyen Âge..., op. cit., p. 100-101.

48. Ibid., $\mathrm{f}^{\circ}$ 94. Ces engins sont des grues à roue et sans doute aussi des sonnettes ou " moutons " (sorte de machines dotées d'un poids coulissant sur des glissières, employées pour battre des pieux ou des pilotis) nécessaires pour l'installation d'échafaudages dans le fossé. 
Figure 2 - Engin, vers 1570, Nantes, utilisé pour la construction d'un pont (dans J.-P. Leguay, « Les ouvriers des chantiers de construction nantais... ", op. cit., p. 12-13)

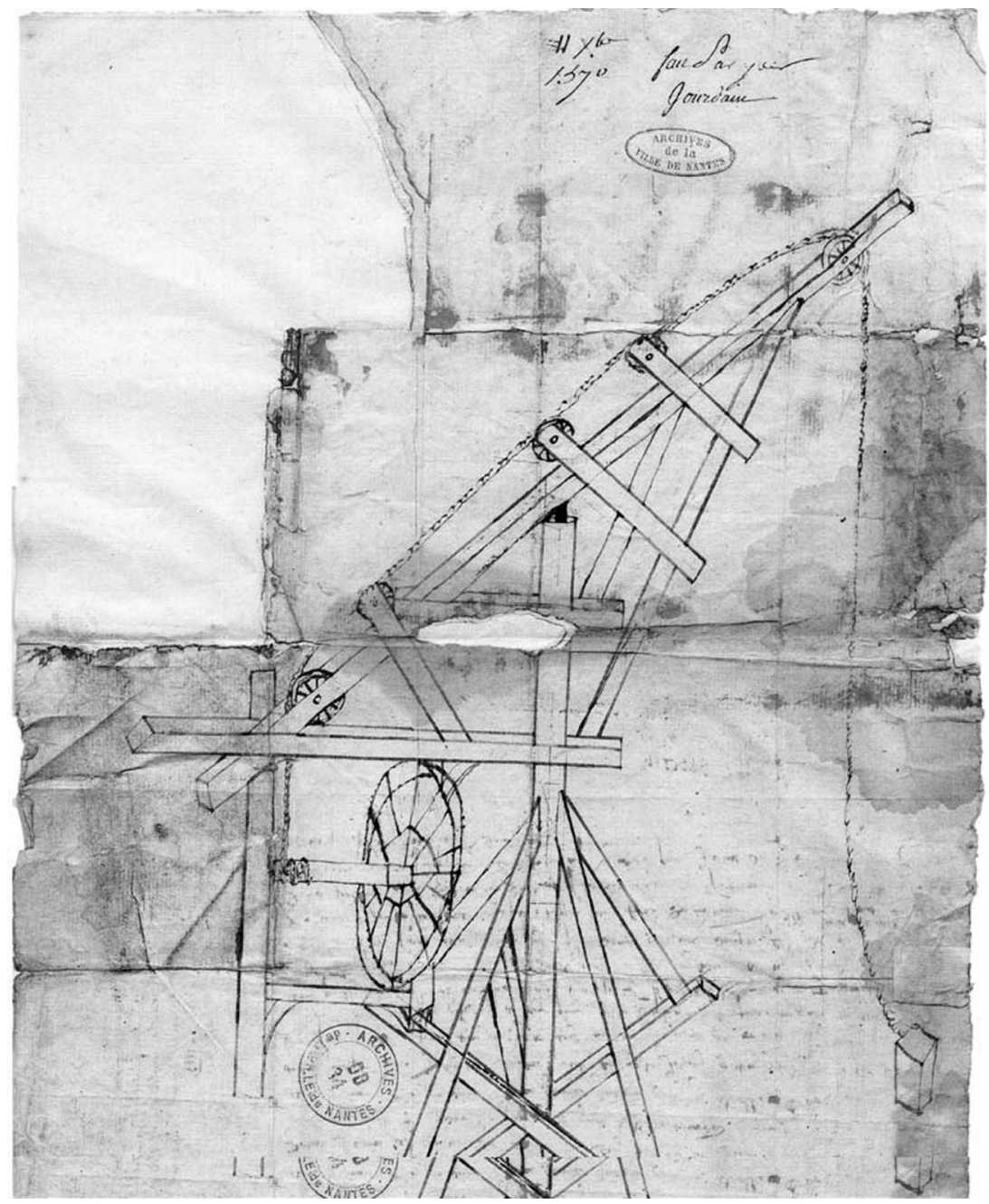

Creuser, recreuser les fossés, nettoyer les abords de la muraille

L'une des tâches importantes de ce chantier consiste à curer les fossés, lesquels se sont remplis au fil des années de terre et de pierres et, on imagine, de toutes sortes de détritus abandonnés ici ou là, favorisant la multiplication des poches d'eaux stagnantes; autant d'éléments qui peuvent empêcher l'eau de s'écouler correctement. Nombreux sont les ouvriers préposés pendant des journées entières à " tirez la pierre, groys et eau des 
fossez ${ }^{49}$ ". Ainsi, Jehan Restineau, perrier, " a perroyé esdits fossez près le portal Saint Aulbin au droit de l'eglise de monseigneur Saint Sauveur ${ }^{50}$ " tandis que cinq autres perriers durant " seix jours [...] ont perroyé devers le portal Saint Nicholas pour tyrez de la pierre par la breche d'entre ledit portal Saint Nicholas et le portau Leonnays ${ }^{51}$ ".

\section{Construire, réparer}

Les comptes prévoient de réparer les fondations de certains murs et dans certains cas d'engager la reconstruction de parties entières de l'enceinte : par exemple, "de faire un pan de muraille es fossés de la ville ${ }^{52}$ ", " de parfere la maczonnerie de la breche d'entre le portau Leonnays et le portau Saint Nicholas tant de machecolleiz que d'autre maczonnerie laquelle breche autant autreffoiz a esté commancée par les maczons de ladite ville ${ }^{53}$ " ou bien encore " de faire ung pan de muraille es fossez de ladite ville d'Angiers joignant ung autre pan de mur que ledit Belin a nagueres fait esdits fossez au portal Saint Aubin suyvant jucques à l'endroit de l'eglise de Saint Sauveur contenant ledit pan de muraille deux cens quatre vigns piez de long ou environ et de large par bas sur le nayff de quatre piez et par hault de troys piez ou environ en bataison de la haulteur dudit autre pan de mur dessusdit qu'il a fait; lequel pan de muraile il joindra et lyera avecques ledit autre $\operatorname{pan}^{54 " .}$

Cette diversité des travaux engagés suppose d'avoir recours à différents corps de métier : trois groupes distincts peuvent être identifiés.

\section{Les moyens humains et techniques}

\section{Effectifs et corps de métiers présents sur le chantier}

Un premier groupe d'ouvriers est constitué de toute une cohorte d'individus que l'on pourrait qualifier de manœuvres. Au sein de cet ensemble, il est toutefois tentant de différencier les ouvriers non spécialisés, uniquement employés pour la force physique qu'ils sont en mesure de fournir, de ceux qui, au fil du temps, ont acquis un petit niveau de spécialisation. Ainsi, on distinguera les hommes qui sont là pour vider les fossés, transporter dans des hottes, dans des paniers et sur des civières les résidus de démolition, la pierre mêlée à la terre, de ceux qui assistent les maçons et les charpentiers en leur apportant, par exemple, des pierres choisies

49. Ibid., $\mathrm{f}^{\circ} 32 \mathrm{v}^{\circ}$ et $\mathrm{f}^{\circ} 83$. La groie est du limon mais le mot signifie ici de la boue et du remblai.

50. Ibid., $\mathrm{f}^{\circ}$ 22. Cette localisation désigne la proximité de la porte Saint-Jean-Baptiste au nord de celle de Saint-Aubin mais qui n'est pas mentionnée ici car elle était condamnée à cette époque.

51. Ibid., $\mathrm{f}^{\circ} 75 \mathrm{v}^{\circ}$.

52. Ibid., $\mathrm{f}^{\circ} 16$.

53. Ibid., $\mathrm{f}^{\circ} 20$.

54. Ibid., $\mathrm{f}^{\circ} 16$. 
et parfois dégrossies par leurs soins, du bois et tous les outils dont ils ont besoin; ils peuvent aussi préparer le mortier ${ }^{55}$. Des miniatures représentent des chantiers montrant ces travailleurs qui transportent parfois de lourdes charges et grimpent sur des échafaudages pas toujours très stables $^{56}$. Parmi ces manœuvres, on trouve les brassiers que l'on appelle dans le compte les " gens ou oupvriers loués ${ }^{57}$ ", les " serviteurs de maczons $^{58}$ ", les " hommes de bras ${ }^{59}$ ", tel ce Jehan Sigourgneau qui est payé sept sous six deniers "pour cinq journés qu'il a besongné esdits fossez ceste presente sepmaine ${ }^{60}$ ». En moyenne, on estime qu'il y a entre un ou deux manœuvres pour un maçon ou un charpentier mais des équipes plus nombreuses peuvent être mobilisées quand l'ampleur des tâches à accomplir l'impose. C'est le cas, par exemple, lorsqu'il faut creuser entièrement des douves ou ouvrir une carrière.

Dans ce groupe, se trouvent ceux que l'on dénomme les perriers et les bessons. Si les premiers sont des spécialistes de l'extraction de la pierre, ils sont carriers tels Pierre Chenede, Pierre Frocquet et Guillaume Cayrau qui ont gagné vingt-sept sous tournois " pour chacun troys jours qu'ilz ont perroyé ceste sepmaine au portal Saint Nicholas qui est au pris de troys soulz par jour ${ }^{61}$ "-, les seconds, davantage pionniers ou terrassiers, sont cantonnés au travail de la terre. Yvonnet Lemoulnier, Jean Lejars et Noël Richart qui ont promis de "fere et chercher les fondemens des fossez de ladite ville pour faire muraille ${ }^{62}$ " touchent ainsi la somme de cinquante livres tournois et une pippe de vin pour ce travail.

Le second groupe, constitué d'individus plus qualifiés mais moins nombreux, s'organise autour des maçons et des charpentiers. Dans certains cas, les maçons n'étaient d'ailleurs pas uniquement cantonnés à des tâches de maçonnerie et pouvaient faire office de conducteur de travaux, voire de maître d'œuvre ${ }^{63}$. Appréciés pour leur savoir-faire parfois très technique, Thebaut Belin " maczon demourant à Laval ", Jean Roger et Jean Lore " aussi maczons de la parroaise de Soucelle " s'engagent ainsi à " faire de

55. Cette distinction n'est pas propre à ce chantier, comme l'atteste l'article de PINTO, Giuliano, "Les rémunérations des salariés du bâtiment (Italie, XIII ${ }^{\mathrm{e}} \mathrm{XV} \mathrm{e}^{\mathrm{e}}$ siècles) : les critères d'évaluation ", dans Beck Patrice, Bernardi, Philippe, FELlER, Laurent (dir.), Rémunérer le travail au Moyen Âge. Pour une histoire sociale du salariat, Paris, A. et J. Picard, 2014, p. 315 .

56. BAUd, Anne, BERNARDI, Philippe, HARTMANN-VIRnICH, Andreas, et al., L'échafaudage dans le chantier médiéval, Lyon, DARA, n 13, 1996.

57. Par exemple, Bib. mun. d'Angers, ms 1148 (946), $\mathrm{f}^{\circ} 30 \mathrm{v}^{\circ}$.

58. Par exemple, Ibidem, $\mathrm{f}^{\circ} 60 \mathrm{v}^{\circ}$ ou f $80 \mathrm{v}^{\circ}$.

59. Ibid., $\mathrm{f}^{\circ} 57$.

60. Ibid., $\mathrm{f}^{\circ} 56$.

61. Ibid., $\mathrm{f}^{\circ} 78$.

62. Ibid., $\mathrm{f}^{\circ} 14$.

63. Sur le sujet, voir CHAPElot, Odette (dir.), Du projet au chantier. Maîtres d'ouvrage et maîtres d'œuvre aux XIV -XVI siècles, Paris, Éditions de l'École des Hautes Études en Sciences Sociales, 2001. 
la main seulement ung pan de muraille ${ }^{64}$ ». Les charpentiers sont, quant à eux, engagés pour mettre au point des installations permettant d'extraire et d'acheminer plus facilement la pierre et la terre tirées des fossés ainsi que divers échafaudages. Guillaume Guerineau et Guillaume Mousset reçoivent quant à eux dix livres " sur ce que leur peut ou pourra estre deu de la faczon des deux engins qu'ilz font pour tirez la pierre et grois desdits fossez ${ }^{65}$ ". La mise au point de ces installations nécessite le recours au service d'un cordier, à l'instar d'Yvonnet Masnet qui a été payé cent sous " par ledit Perier pour des cordages " dont on précise en marge qu'ils devront être rendus à la ville, ainsi que d'un canonnier, nommé André Aubert, qui est rémunéré quatorze livres quinze sous pour les poulies de l'engin.

Le troisième groupe rassemble les professions en marge du chantier mais nécessaires à la bonne conduite de ce dernier. L'approvisionnement en matériaux est assuré par les nautoniers, ces marchands qui fréquentent la rivière, et les charretiers qui transportent les matériaux par terre sur les différents chantiers et entre les lieux de production ou d'arrivage et les chantiers. Jean Nillesen et Colas Godart, " notonniers ", perçoivent ainsi sept livres un sous quatre deniers pour 212 tombereaux de sablon " qu'ilz ont baillé et livré au port Lynier ${ }^{66}$ ". La mise en œuvre des travaux nécessite également la présence de " chaussumiers " pour produire et livrer la chaux. Jean Aubry est payé vingt-trois livres quinze sous pour neuf muids de chaux " qu'il a baillez pour la reparacion desdits fossés ${ }^{67}$ ". Globalement, le compte de Jean Perier fournit peu de renseignements sur ces travailleurs mais cela n'a rien d'étrange puisqu'il n'entre pas dans ses attributions de le faire.

Les noms de famille laissent toutefois penser que le recrutement des travailleurs est plutôt local, même si la présence, par exemple, d'un Dyago Ferrando, Jean de Chinon, Jean de Bourges, Jean de Paris ou André Savoie peut laisser entendre que certains d'entre eux viennent de régions parfois fort éloignées de l'Anjou. Jean-Pierre Leguay rappelle d'ailleurs que plusieurs études ont bien montré l'existence d'une mobilité des travailleurs des chantiers : dans certaines contrées, telles que le Piémont, l'Angleterre, l'Allemagne, la Suisse ou la Savoie, les itinérants peuvent même atteindre jusqu'à $90 \%$ des effectifs d'un chantier de construction ${ }^{68}$.

La tentation est grande d'essayer de quantifier le nombre d'individus qui travaillent sur ce chantier. Le compte permettrait de le faire car il fournit des listes de noms organisées par métier. Il faut toutefois rester prudent compte tenu de la fréquence de l'homonymie au Moyen Âge et du peu d'informations concernant ces travailleurs; cela rend la mesure précise

64. Ibidem, $\mathrm{f}^{\circ} 11$.

65. Ibid., $\mathrm{f}^{\circ} 32 \mathrm{v}^{\circ}$.

66. Ibid., $\mathrm{f}^{\circ} 32$.

67. Ibid., $\mathrm{f}^{\circ}$ 47. Le mot habituel est chaufournier.

68. LEGUAY Jean-Pierre, «Les ouvriers des chantiers de construction nantais... », op. cit., p. 6. 
impossible. Par ailleurs, les chiffres avancés ne veulent pas dire que tous ces ouvriers étaient en même temps sur le chantier. Ils ne tiennent pas toujours compte non plus des ouvriers intervenant dans le cadre des marchés ni même des corvéables qui ont pu être amenés à venir effectuer diverses tâches comme l'atteste le document suivant, légèrement postérieur au compte de Jean Perier :

"Les commissaires commis et ordonnés par le Roy notre sire à mectre et imposer sur les habitans à sept lieues ${ }^{69}$ à la ronde de la ville d'Angiers le nombre de cent hommes par chacun jour à besoigner es fossez de la ville d'Angiers ainsi que par les lectres du Roy notre dit sire contenant notre commission donnée au Plessis du Parc le XviII jour de fevrier derrenier passé peut plus à plain apparoir aux parroissiens de Joué ${ }^{70}$. Salut, nous vous mandons et expressement enioignons de par le Roy notre dit sire et sur les paines contenues esdites lectres royaulx contenant notre dite commission que vous fournissiez au xxIx ${ }^{\mathrm{e}}$ jour du moys octobre present venant le nombre de XLVI hommes journeliers et bons ouvriers pour besongner esdits fossez et armez de pelles, pics ou tranches lesquelx seront tenuz estre eulx presentez esdits fossez ledit jour avant six heures du matin et y besongnent tout le jour ainsi que par les commis à le faire leur savoir ordonné et garder qu'il n'y ait faulte sur paine d'estre repputer rebelles et desobbeissans au Roy notre dit sire. Donné à Angiers soubz noz saingns manuelz le xxvI jour de septembre l'an mil cccc soixante dix huit ${ }^{71}$."

Quantitativement, les manœuvres sont les plus nombreux avec une soixantaine de "gens loués ", plus de soixante-dix bessons et une bonne trentaine de perriers.

Avec deux charpentiers et cinq maçons, les ouvriers spécialisés sont là pour dispenser leur savoir et sans doute superviser les travaux. Et lorsqu'ils s'adonnent à leur métier, ils sont secondés par des hommes dont la tâche est bien de les "servir ". Guillaume Cornu, Pierre Drouet et André Jouyn gagnent ainsi trente sous tournois chacun pour avoir passé " V jours de ceste sepmaine à servir les maczons ${ }^{72}$ ». L'approvisionnement des différents sites du chantier de 1478 se fait grâce aux services d'à peine une vingtaine de nautoniers et charretiers qui apportent tous les matériaux nécessaires aux réparations ${ }^{73}$; ces derniers sont acheminés, par exemple, du port Linier à la porte Saint-Aubin. Sur le chantier, se trouve également une quarantaine d'ouvriers dont la tâche est de préparer la chaux : ils versent de l'eau sur le calcaire pendant des heures afin de «l'estaindre » puis l'acheminent au

69. Une lieue d'Anjou correspond à environ 4,7 kilomètres, donc sept lieues font environ 33 kilomètres.

70. JouÉ, Maine-et-Loire, arr. Angers, cant. Thouarcé, comm. Valanjou.

71. Arch. dép. de Maine-et-Loire, 3E 21, 1478, communauté de Joué, travail aux fossés de la ville d'Angers, 1 feuille volante.

72. Bib. mun. d'Angers, ms 1148 (946), $\mathrm{f}^{\circ} 66 \mathrm{v}^{\circ}$.

73. À titre de comparaison, lire LEGUAY, Jean-Pierre, "L'approvisionnement des chantiers bretons en matériaux de construction aux XIV et XV e siècles ", dans CHAPELOT, Odette, BEnort, Paul (dir.), Pierre et métal dans le bâtiment au Moyen Âge, Paris, Éditions de l'École des Hautes Études en Sciences Sociales, 1985, p. 27-79. 
pied de l'enceinte. Les réfections ont également nécessité la contribution de deux cordiers - Yvon Masnet est rétribué cent sous pour les "cables et les cordaiges qu'il a baillez pour les engins desdits fossés ${ }^{74}{ }-$, d'un canonnier qui fournit des poulies pour l'engin et de quelques artisans "métallurgistes " qui fournissent par centaines des clous, vis et pelles ferrées.

À la marge des travaux, le compte permet également de mettre en lumière la présence d'individus dont la tâche est d'assurer le service de l'eau et du vin sur le chantier (une petite vingtaine de noms) mais aussi de soigneurs auxquels incombe le devoir de s'occuper des blessés, d'un boucher qui a fourni une peau de mouton pour couvrir un accidenté et d'un cordonnier qui a confectionné des chaussures pour certains travailleurs. En l'état, ce sont donc quelque 250-280 personnes qui ont travaillé sur ce chantier de 1478, auxquelles, nous le rappelons, il faudrait ajouter les corvéables et les travailleurs prévus dans les marchés. Au total, ces travaux ont nécessité la présence de quelques centaines de personnes, ce qui représente un chiffre non négligeable si on le rapporte à l'ensemble de la population angevine, estimée à quelque 10000 à 12000 habitants ${ }^{75}$.

En matière d'économie du chantier, ce compte offre d'intéressantes informations sur les salaires qui sont versés à chaque profession comme sur le prix des matériaux et des outils; il appartiendrait de traiter ces aspects dans un autre article tant il y aurait à dire. Simplement, on pourra remarquer que les différences de statut entre les ouvriers se retrouvent dans les rémunérations. En outre, tous les salaires ne sont pas versés de la même manière : par exemple, certains sont payés à la journée, là où d'autres sont rémunérés pour les fournitures acheminées ou pour la tâche effectuée ${ }^{76}$.

Ainsi, en moyenne, un perrier gagne environ 36 deniers par jour et un besson aux alentours de $25^{77}$, des rémunérations proches de celles relevées par Jean-Pierre Leguay sur un chantier à Nantes dans la première moitié $\mathrm{du} \mathrm{XV}^{\mathrm{e}}$ siècle $^{78}$. De tels niveaux de salaire laissent penser que, dans leur

74. Ibid. $\mathrm{f}^{\circ} 47$.

75. LEBRUn, François, "Angers sous l'Ancien Régime : introduction à l'étude démographique de la population ", Annales de Bretagne et des pays de l'Ouest, 1974, p. 151-166.

76. En lien avec le sujet, voir BECK Patrice, « Notes sur l'embauche et la rémunération du travail dans les métiers du bâtiment en Bourgogne à la fin du Moyen Âge : le chantier de construction des pressoirs ducaux de Chenôve près de Dijon (1400-1404) ", dans BECK, Patrice, BERNARDI, Philippe et FelLER Laurent (dir.), Rémunérer le travail au Moyen Âge..., op. cit., p. 296-300 et BAULANT, Micheline, " Le salaire des ouvriers du bâtiment à Paris de 1400 à 1726 ", Annales ESC, 1971-2, p. 463-483.

77. Dans le registre, on trouve ainsi des listes de noms d'ouvriers avec des comptes globaux. Par exemple, "Somme vI bessons dudit jour à II s. I d. par jour vallent XII s. VI d. tournois " $\left(\mathrm{f}^{\circ} 26 \mathrm{v}^{\circ}\right)$ ou "Somme IX perriers à III $\mathrm{s}$. tournois par jour vallent XXVII $\mathrm{s}$. tournois" ( $\left.\mathrm{f}^{\circ} 27\right)$.

78. L'auteur propose l'échelle de salaires suivante : 40 à 50 deniers par jour pour un maître; 24 à 36 deniers par jour pour un simple ouvrier maçon, charpentier, couvreur; 20 à 24 deniers par jour pour un manœuvre; 10 à 15 deniers par jour pour un adolescent ou une femme. 
majorité, les ouvriers ne gagnent pas beaucoup et que leur condition précaire leur permet de subvenir tout juste à leurs besoins essentiels. À cette occasion, on remarquera que s'il est fait référence à un service de l'eau à destination des ouvriers, en revanche il n'est pas question de distribution de repas. Comme le souligne très bien Sandrine Victor, " cette question de savoir si les ouvriers étaient nourris ou non sur les chantiers peut revêtir un caractère important si on prend en considération le fait qu'un ouvrier pouvait dépenser $60 \%$ de son salaire pour se nourrir ${ }^{79} "$.

Si la rémunération à la journée concerne très majoritairement les perriers, les bessons et les simples manœuvres, ils n'ont toutefois pas le monopole de ce traitement. Ainsi, Simon Menart, charretier, gagne trois sous neuf deniers "pour avoir mené troys jours de sablon du port Lynier au portal Saint Aulbin à la raison de XV deniers par jour ${ }^{80}$ ", tandis que Guillaume Guerineau, charpentier, " a esté payé par ledit Perier par le commandement et ordennance de mondit sieur le maire la somme de cinquante soulz tournois pour doze journées qu'il a esté luy et ses varletz à foncez la roe dudit engin du portal Saint Aulbin et faire ung tour tout neusf à ladite roe et a fourny de la piece de boais à fere ledit tour ${ }^{81}$ ". De même, ce type de rémunération n'exclut pas des différences au sein d'un même groupe d'ouvriers. On a l'exemple de ces "gens à louez qui ont besongné esdits fossez ceste sepmaine qui commancza le lundi $\mathrm{XX}^{\mathrm{e}}$ jour dudit moais d'apvril chacun par cinq jours ${ }^{82}$ ". Ils sont seize au total, douze d'entre eux sont payés sept sous six deniers tandis que Jean de Paris gagne douze sous six deniers, André Savoye dix sous; Pasquier Romigny quinze sous, et Herbert Cherhoste vingt sous mais, précise-t-on, " pour deux sepmaines ». Le registre n'explique pas ces différences de salaire. On peut avancer quelques hypothèses pour expliquer ces écarts : des particularités dans le travail accompli, des différences de compétences, de responsabilités et de rang entre les ouvriers.

Certains travailleurs sont rémunérés pour les marchandises qu'ils fournissent, les sommes variant selon le type de fournitures (matériaux plus ou moins coûteux, fabrication plus ou moins élaborée), les distances parcourues pour acheminer les chargements et également la facilité avec laquelle ils peuvent rejoindre les chantiers. Ainsi, Lucas Godart « notonnier a esté paié par ledit Perier [...] la somme de quatorze soulz tournois pour Xxi tumbelerée de sablon prins de luy pour lesdits ouvraiges ${ }^{83}$ " tandis que Gervaise Busson et Simon Menart, charretiers, ont reçu « pour le charroy de XXI tumbelerée de sablon qu'ilz ont mené du port Lynier au portal Saint

79. . VICTOR, Sandrine, "Les formes de salaires sur les chantiers de constructions : l'exemple de Gérone au bas Moyen Âge ", dans BEcK, Patrice, BERnARDI, Philippe et FELLER Laurent (dir.), Rémunérer le travail au Moyen Âge..., op. cit., p. 260. L'auteure explique d'ailleurs que " le $\mathrm{xv}^{\mathrm{e}}$ siècle voit disparaître la tradition du repas offert chaque jour par l'employeur".

80. Bib. mun. d'Angers, ms 1148 (946), $\mathrm{f}^{\circ} 37 \mathrm{v}^{\circ}$.

81. Ibidem, $\mathrm{f}^{\circ} 56 \mathrm{v}^{\circ}$.

82. Ibid., $\mathrm{f}^{\circ} 37$.

83. Ibid., $\mathrm{f}^{\circ} 31 \mathrm{v}^{\circ}$. 
Aulbin pour les ouvres de ladite ville d'Angiers ${ }^{84}$ " la somme de vingt-sept sous trois deniers. Si les matériaux sont souvent peu onéreux, en revanche leur transport peut faire considérablement augmenter la facture.

D’autres ouvriers, enfin, peuvent être rémunérés à la tâche. Cette forme de rémunération est utilisée aussi bien pour les activités en lien direct avec la gestion du chantier que pour l'acheminement des matériaux ou la confection d'ouvrages et d'outils. Ainsi, Jean Lemoulnier gagne vingt sous "pour avoir vuidé les eaux desdiz fosés ${ }^{85}$ ", Jean Theon trois sous "pour avoir cassé les grosses pierres de la tasche Restineau ${ }^{86}$ " et Henry Mesleart cinquante sous " pour avoir appareillé les arches du pré de la Savate et mis le boys necessaires par marché fait avecques luy ${ }^{87}$ ". Quant à savoir ce qui motive le recours à tel type de rémunération plutôt qu'à tel autre, les sources sont muettes sur le sujet.

Pour le moment, comme nous venons de le voir, les individus travaillant à la réparation de l'enceinte sont des personnes qui s'engagent librement dans cette entreprise, à l'exception des corvéables dont on sait qu'ils sont présents sur ce type de chantiers ${ }^{88}$; ils mettent au service de la ville, contre salaire, leur savoir-faire ou plus modestement leurs bras. Mais tel n'est pas toujours le cas.

En effet, quinze articles de dépense étalés entre le 27 juin et le 12 septembre permettent de constater que travaillent également sur ce chantier des hommes contraints par la justice. Les passages dans le compte sont sans équivoque quant à leur statut d'individu ayant eu des démêlés avec l'institution judiciaire. Ainsi, maître Alexandre Chalmeaux est payé trentesix sous trois deniers tournois " pour la despence de Estienne de Besches, Michel Treguy, Benoist Vysault, Robin Pays, Yvonnet Pierre, Jacquet Loyselier, Jehan Hemont et Regné Guillou qui ont esté ceste sepmaine à besongner esdits fosés et ont eu chacun une paire de soulliers que ledit mestre Alixandre leur a baillez et delivrez ${ }^{89}$ ", cinquante et un sous quatre deniers tournois " pour XIV paires de soulliers qu'il a fait baillez à XIV jeunes hommes qui ont esté prins par justice pour besongnez esdits fosez lesquelx estoint oyseux ${ }^{90}$ " puis il perçoit quatre livres deux sous " pour la despence de XXIV jeunes hommes qui ont besongné esdits fossez ceste sepmaine cha-

84. Ibid., $\mathrm{f}^{\circ} 32$. Le sablon est un sable fin utilisé en construction.

85. Ibid., $\mathrm{f}^{\circ} 31 \mathrm{v}^{\circ}$.

86. Ibid., $\mathrm{f}^{\circ} 44 \mathrm{v}^{\circ}$. J. Theon est considéré comme le remplaçant du carrier J. Restineau.

87. Ibid. $\mathrm{f}^{\circ}$ 84. Ces arches qui prolongent l'enceinte côté rive droite jusqu'au bras principal de la Maine n'ont été construites qu'au xve siècle. Le bois a été utilisé pour les pieux de fondation.

88. Les délibérations laissent entrevoir qu'il y a toute une frange de la population angevine qui était bien contrainte de donner de son temps pour l'entretien des fossés et du mur de l'enceinte; voir par exemple, Arch. mun. d'Angers, BB 1, f $\mathrm{f}^{\circ} 5 \mathrm{v}^{\circ}$ : "Aussi a esté appoincté que les deffaillans des fossez de la ville seront mis à besongner aux cannaulx de la ville qui sont empeschez pour y besongner comme il leur sera ordonné " (15 décembre 1479).

89. Bib. mun. d'Angers, ms 1148 (946), f 65.

90. Ibidem, $\mathrm{f}^{\circ} 68 \mathrm{v}^{\circ}, 4$ juillet. "Oyseux " signifie sans travail. 
cun par seix jours lesquelx estoint oyseux querrant leur pain par la ville d'Angiers ${ }^{91}$ " et vingt-sept sous dix deniers " pour seix plaz de boys, deux potz de terre, seix terrins, trois paire de soulliers neuff carreleurés de cuyr le tout baillé par ledit Chalmeaux ausdits XxIV jeunes hommes oyseux qui ont esté mis à besongner esdits fosés par l'ordennance de mesdits sieurs de la justice ${ }^{92}$ ".

Colas Tardif, " carreleur" de son état, reçoit quant à lui seize sous trois deniers "pour avoir carrelé treze paires de soulliers par les gens oyseux par le commandement de mondit sieur le maire lequel Tardif a fourny de cuyr ${ }^{93}$ " tandis qu'Ysabeau la Cerisiere touche soixante-trois sous six deniers "pour la despence de XxIv hommes qui ont esté mis à besongner esdits fosez par justice lesquelx estoient oyseulx et ont besongné ceste sepmaine chacun par cinq jours et lesquelx sont aux despens sur les deniers de la ville ${ }^{94}$ " et Perrine la Girarde sept sous six deniers " pour avoir blanchy et relavé le linge de l'aumosnerie de Brechigné ${ }^{95}$ ou couschent xxIIII compaignons qui besongnent esdits fosez par l'ordennance de mondit sieur le maire et de messieurs de la ville ${ }^{96}$ ".

Enfin, Geffroy Franczois touche à huit reprises des sommes d'argent : il gagne quatre sous " pour la despence de Estienne Estable qui a esté mi à besongner esdits fossez pour quatre jours ${ }^{97}$ "; trente sous " pour la despence de troys prinsonniers qui ont besongné ceste sepmaine esdits fossez chacun par seix jours et aussi pour la despence de deux autres prinsonniers qui ont esté par troys jours en prinson ou portal Saint Aubin ${ }^{98}$ "; vingt et un sous "pour la despence des trois prinsonniers qui ont esté mis à besongner esdits fossez pour les jours de dimanche, lundi, mardy, mercredi, jeudi, vendredi et sabmedi ${ }^{99}$ "; quatorze sous " pour la despence de Mathurin Estable et Yvonnet Paris prinsonniers qui ont besongné esdits fosez ceste sepmaine chacun quatre jours et est pour toute leur despence de ceste sepmaine depuis dimanche jucques à demain au soir ${ }^{100 ~}$ "; seize sous " pour la despence de Jehan Saune, Yvonnet Paris et Estienne Figeat qui ont besongné ceste sepmaine esdits fossés savoir est lesdits Saune et Paris chacun par seix jours et ledit Figeat par deux jours lesquelx sont prinsonniers et ont esté mis à besongner esdits fosés par l'ordennance de messieurs du conseil ${ }^{101}$ "; vingt et un sous " pour la despence de Jehan Saune,

91. Ibid., $\mathrm{f}^{\circ} 70 \mathrm{v}^{\circ}, 11$ juillet.

92. Ibid., $\mathrm{f}^{\circ} 71,11$ juillet.

93. Ibid., $\mathrm{f}^{\circ} 69,4$ juillet. " Carreler " signifie ressemeler.

94. Ibid., $\mathrm{f}^{\circ} 69 \mathrm{v}^{\circ}, 4$ juillet.

95. L'aumônerie Saint-Sébastien dans le faubourg Bressigny, sorte d'asile pour pauvres passants, a été fondée en 1360 pour 6 lits. Les capacités d'accueil semblent avoir sensiblement augmenté puisqu'un siècle plus tard, en 1478, ce sont 24 ouvriers qui y sont logés.

96. Bib. mun. d'Angers, ms 1148 (946), f ${ }^{\circ}$ 75, 18 juillet.

97. Ibidem, $\mathrm{f}^{\circ} 70,4$ juillet.

98. Ibid., $\mathrm{f}^{\circ} 76,18$ juillet.

99. Ibid., $\mathrm{f}^{\circ} 79,25$ juillet.

100. Ibid., $\mathrm{f}^{\circ} 89 \mathrm{v}^{\circ}, 14$ août.

101. Ibid., f ${ }^{\circ} 91,22$ août. 
Yvonnet Paris et Jehan Lemerle de dimanche, lundi, mardi, mercredi, jeudi, vendredi et sabmedi lesquelx ont besongné esdits fosés par l'ordennance de justice ${ }^{102}$ "; vingt-cinq sous cinq deniers " pour la despence de Jehan Saune et Yvonnet Paris qui ont besongné esdits fossez ceste sepmaine par ordennance de justice et à Jehanne la Rogiere qui a servy de ayne ceste sepmaine par vi jours seix soulz tournois et audit Yvonnet Paris cinq soulz cinq deniers pour une chemise par le commandement et ordennance de Simonnet Sabart lieutenant de mondit sieur le soubzmaire le $\mathrm{v}^{\mathrm{e}}$ jour dudit moys de septembre l'an dessusdit mil $\mathrm{IIII}^{\mathrm{cc}}$ LXXVIII ${ }^{103}$ " et quatorze sous " pour la despence de Jehan Saune et Yvonnet Paris pour sept jours lesquelx besongnent esdits fosés par ordennance de justice ${ }^{104}$ ".

Visiblement, à l'instar d'Alexandre Chalmeaux ou de Geffroy Franczois, l'entretien de ces repris de justice est une activité qui peut s'avérer rémunératrice. Logés, nourris, blanchis, ils sont également explicitement chaussés et vêtus; cette vingtaine d'hommes dont certains, à l'image d'Yvonnet Paris ou Jean Saune, ont travaillé près d'un mois sans interruption dans les fossés, est prise en charge par la mairie. Des études ont bien montré qu'à la fin du Moyen Âge, le vagabondage des bien portants, que l'on qualifie d'oiseux, est considéré, dans beaucoup d'endroits, comme un délit de justice ${ }^{105}$. Un tel état d'esprit transparaît d'ailleurs nettement dans les coutumes de l'Anjou et du Maine : " compaignon oyseux et vacabont qui n'a de quoy vivre, doit par la justice estre emprisonné et enquis. Et qui ne trouve assez matiere de quoy le pugnir soit banny du pais, si le cas le requiert ${ }^{106}$ ". Ces cas mentionnés dans le compte de Jean Perier mettent en avant l'idée d'une régénération par le travail des faux pauvres et des inactifs; idée que l'on voit poindre un peu partout à la fin du Moyen Âge ${ }^{107}$.

102. Ibid., f ${ }^{\circ} 94,28$ août.

103. Ibid., $\mathrm{f}^{\circ} 97,5$ septembre.

104. Ibid., $\mathrm{f}^{\circ} 98,12$ septembre.

105. Sur la criminalité urbaine, voir, par exemple, LeGUAY, Jean-Pierre, Un réseau urbain au Moyen Âge : les villes du duché de Bretagne aux XIV et XV siècles, Paris, Maloine, 1981, p. 285-297.

106. Beautemps-Baupré, Charles-Jean, Coutumes et institutions de l'Anjou et du Maine antérieures au XVI siècle, Coutumes et styles, Paris, A. Durand et Pedone-Lauriel éditeurs, 1877-1883, t. 4, partie L ( ${ }^{\mathrm{e}}$ moitié du Xve siècle), dix-huitième partie " Des crismes et des pugnicions d'iceulx ", $\S 294$, p. 267. Cette prescription se retrouve dans les autres versions des coutumes, voir t. 1, partie $\mathrm{E}$ (1411), quatrième partie, § 84, p. 434; t. 2, partie F ( $\mathrm{XV}^{\mathrm{e}}$ siècle), première partie, titre IV « Des juges ", $§ 43$, p. $48-49$ et t. 3 , partie I (1463), « La quarte partie ", § 103, p. 264-265.

107. Dans un ordre d'idées proche, on voit encore en 1521 la mairie d'Angers prendre des mesures pour mettre au travail les mendiants : " Aussi ont esté d'avis les dessusdits pour ce qu'il y a en ceste ville et y affaire chacun jour grant nombre de belistres et paouvres quy viennent de XL divers pays et contrées où l'on se meure pour et obvier ad ce et que l'on trouve occasion de les chasser plus aisement hors ladite ville que l'on contraigne lesdits belistres qui pourront besongner à curer les rues de la ville et à besongner aux douves et foussez d'icelle et pour ce faire a esté ordonné et conclud qu'il sera fait aux despens de ladite ville jusques à deux douzaines de hostes et deux douzaines de cyvieres pour faire ledit nectoyement et curement " (Arch. mun. d'Angers, BB 17, $\mathrm{f}^{\circ}$ 141, délibérations du mardi premier octobre 1521). 
On le sait, au XVI ${ }^{\mathrm{e}}$ siècle la législation sur le sujet sera des plus sévères ${ }^{108}$. Là où l'idéal chrétien de charité est fortement recommandé à l'égard des plus faibles, des pauvres et des oisifs, les autorités urbaines choisissent parfois d'adopter une politique ouvertement répressive. En ce qui concerne le cas concret de ces Angevins, nous n'en savons pas davantage. En effet, il n'y a pas d'archives judiciaires à proprement parler connues pour la mairie d'Angers car ces questions sont abordées dans les registres de délibérations du corps de ville, lesquels commencent malheureusement plus tard, en $1479^{109}$. Qu'en est-il des éventuels délits de ces hommes, des éventuelles condamnations qui les ont frappés? La question reste entière.

$\mathrm{Si}$, comme nous venons de le voir, le profil des travailleurs est varié, il en va de même du matériel et des matériaux nécessaires à la mise en œuvre des travaux.

\section{Matériel et matériaux}

Le chantier nécessite de gros moyens pour que soit menée dans de bonnes conditions la réfection des fossés et des murs de l'enceinte : des matériaux bruts et transformés (sable, pierre, chaux, mortier) mais également du petit et gros matériel ouvragé.

\section{Des matériaux bruts et transformés}

Le chantier consomme des quantités importantes de sable qualifié de "sablon" dans le compte ${ }^{110}$. Robin et Gervaise Busson, charretiers, touchent ainsi soixante sous " pour la voiture de 83 tours de sablon qu'ilz ont mené du port Lynier au portal Saint Aulbin pour les euvres de ladite ville d'Angiers ". En lui-même, le sable ne coûte pas cher, en revanche, son transport est onéreux. Il y a quelques difficultés à appréhender les volumes de sable qui ont été acheminés sur le chantier en raison des différentes unités de mesure qui sont employées : le compte fait état de 1300 tombereaux de sablon (sachant qu'un tombereau équivaut à environ $1 \mathrm{~m}^{3}$ ), de 327 tours de sablon et de 258 pipes de sablon (sachant qu'une pipe équivaut à 445 litres environ). Il faut également des pierres mais la quantité n'en est pas précisée dans le compte, ce qui s'explique très bien car celles-ci sont pour beaucoup extraites ou récupérées sur place. Elles sont utilisées

108. LEGUAY, Jean-Pierre, "Les ouvriers des chantiers de construction nantais... ", op. cit., p. 8. ID., Un réseau urbain..., op. cit., p. 295-297.

109. Les lettres patentes de 1475 qui établissent le corps de ville confèrent à la municipalité des prérogatives de justice importantes que, dix ans plus tard, Charles VIII a considérablement amoindries. Pour davantage de détails, voir ROBERT, René (éd.), Recueil des privilèges de la ville et mairie d'Angers, Angers, 1748; VARANGOT, Jeanne, Les institutions municipales d'Angers de 1474 à 1584, thèse de l'École nationale des chartes, Paris, 1932, p. 129-149; MARTIN, Xavier, L'administration municipale d'Angers à la fin du XVI et au début du XVII siècle, thèse de doctorat de droit, université de Paris, 1973, 5 tomes.

110. En l'état, ce registre ne permet malheureusement pas de dresser une carte précise des lieux d'approvisionnement en matériaux venant de l'extérieur d'Angers. 
pour réparer le mur d'enceinte, ce qu'atteste la référence aux " pierres à maczonner ${ }^{111} "$.

Les ouvriers ont aussi besoin de chaux ${ }^{112}$. Guillemin Augirart " pour seix jours de ceste sepmaine qu'il a servy à tyrez l'eaue pour estaindre la chaux $^{113}$ " est payé 6 sous tournois et Robin Desmazieres touche quant à lui « XL s. pour deux grans cuviers et quatre petiz à mecptre l'eau pour estaindre la chaux de ladite ville d'Angiers ${ }^{114}$ ". En termes de volume, le registre fait état de l'utilisation d'environ $150 \mathrm{~m}^{3}$ de chaux, soit 91 muids et 2 tombereaux. Une fois le sable et la chaux acheminés sur place, les ouvriers peuvent procéder au mélange des deux afin d'obtenir du mortier, nécessaire en grande quantité pour tous les travaux de maçonnerie (sceller les pierres, faire les joints...). Macé Aougstin, Jean Oustin, Jean Ferron, Jean Cherrier et Guillaume Savoie sont ainsi payé deux sous tournois par jour pour avoir fait " le mortier et serv[i] les maczons durant IIII jours ${ }^{115}$ ".

Il a également fallu acheminer du bois, utile à la construction des échafaudages et des engins. Jean Herrault gagne quinze sous pour " deux charretées de perches pour faire les chauffault des maczons qui besongnent esdits fossez ${ }^{116}$ ". Quant à Gervaise Vrigne, il livre " doze picquetz pour les perriers au pris de cinq soulz tournois la piece ${ }^{117}$ ", pendant que Guillaume Guerineau charpentier est payé " dix livres tournois sur le bois et faczon des deux engins ${ }^{118}$ ".

\section{Du gros matériel}

Le compte de Jean Perier fait état de la présence aux abords du chantier de deux engins, un grand et un petit ${ }^{119}$. Raoulet Audouyn ainsi que Guillaume Mouset, Henry Mesleart, Jean Hamonneau et Guillaume Guerineau ont d'ailleurs reçu quelques sous pour aller "à Rochefort veoyr l'engin dudit lieu pour en faire ung au patron ${ }^{120}$ ". Après que le modèle ait été établi, la comptabilité fait état de Guillaume Guerineau charpentier de son état qui a reçu cinquante sous " pour quinze journés qu'il a été luy et ses gens passés à remuez le petit engin et mis près le grant engin et pour trois picquetz neusfs qu'il a faiz et autres choses necessaires pour lesdits engins $^{121}$ ". On sait par ailleurs qu'il y a eu un "blecié à l'engin des douves

111. Bib. mun. d'Angers, ms 1148 (946), f 93.

112. La chaux vive est le produit direct de la calcination du calcaire. Éteinte avec de l'eau, la chaux aérienne peut ensuite être mélangée avec du sable pour donner du mortier.

113. Bib. mun. d'Angers, ms 1148 (946), f $51 \mathrm{v}^{\circ}$.

114. Ibidem, $\mathrm{f}^{\circ} 62 \mathrm{v}^{\circ}$.

115. Ibid., $\mathrm{f}^{\circ} 63 \mathrm{v}^{\circ}$.

116. Ibid., $\mathrm{f}^{\circ} 40 \mathrm{v}^{\circ}$.

117. Ibid., $\mathrm{f}^{\circ} 54$.

118. Ibid., $\mathrm{f}^{\circ} 40 \mathrm{v}^{\circ}$.

119. Voir, par exemple, Ibid., f $\mathrm{f}^{\circ} 40 \mathrm{v}^{\circ}, \mathrm{f}^{\circ} 54 \mathrm{v}^{\circ}, \mathrm{f}^{\circ} 90 \ldots$

120. Ibid., $\mathrm{f}^{\circ} 68 \mathrm{v}^{\circ}$.

121. Ibid., $\mathrm{f}^{\circ} 94$. 
du portal Saint Aulbin ${ }^{122}$ ". De ces informations, il est possible de déduire que les engins peuvent être utilisés séparément ou bien ensemble et, qu'à un moment, ils étaient tous les deux placés près des fossés de la porte Saint-Aubin.

Les engins en question renvoient en fait à des machines, sorte de grues munies d'une roue, de cordes et de poulies, ce qu'atteste très bien la comptabilité de Perier. Gervaise Vrigne reçoit douze livres seize sous huit deniers " pour 247 livres de fer et cent chevilles de fer pour ferrez l'engin qui a été fait pour tirez la pierre et groays des fosez et pour l'acier qu'il a mis aux cotez et pivotz dudit engin ", tandis que Guillaume Guerineau est payé cinquante sous tournois " pour doze journées qu'il a esté luy et ses valetz à foncez la roe dudit engin du portal Saint Aulbin et faire ung tour tout neusf à ladite roe et a fourny de la piece de boais à fere ledit tour ${ }^{123}$ ".

Le but de telles installations est de permettre aux hommes de déplacer des charges lourdes. Ces deux machines sont d'ailleurs suffisamment importantes pour qu'il soit précisé, en marge, que " soient rendues à la ville lesdites polyes ${ }^{124}$ " et que " soit rendu ledit engin à la ville [lequel] est en l'eglise Saint Sauveur ${ }^{125}$ ".

\section{De l'outillage et de la quincaillerie}

Le registre fait état de la nécessité pour les ouvriers d'avoir à leur disposition des fournitures et des outils de toutes sortes. Ce sont par dizaines que se comptent les chevilles, les clous, les crochets, les pointes, les poulies, les ferrures ${ }^{126}$ et les câbles. Les commandes passées sont précises, comme l'attestent ces articles dans lesquels il est demandé à Gervaise Vrigne de livrer des " chevilles de fer", des "grosses chevilles pesans dix et huyt livres fer oupvré ${ }^{127}$ ", tandis que Pierre Bougeri doit fournir, entre autres choses, des " chevilles à teste ronde ${ }^{128}$ ". Plus marginal mais néanmoins essentiel, les ouvriers ont également besoin d'" une livre savon, deux livres sayn pour gressez ledit engin ${ }^{129}$ ".

Les outils nécessaires pour mener à bien les travaux sont également nombreux : des pelles, des marteaux à casser les pierres et des "bec-

122. Ibid., $\mathrm{f}^{\circ} 48 \mathrm{v}^{\circ}$.

123. Ibid., $\mathrm{f}^{\circ} 56 \mathrm{v}^{\circ}$.

124. Ibid., $\mathrm{f}^{\circ} 83 \mathrm{v}^{\circ}$.

125. Ibid., $\mathrm{f}^{\circ} 32 \mathrm{v}^{\circ}$.

126. Ibid., $\mathrm{f}^{\circ} 71 \mathrm{v}^{\circ}$ : "À Gervaise Vrigne a esté paié par ledit Perier commissaire dessusdit par le commandement et ordennance de mondit sieur le maire la somme de neuff livres tournois qui luy ont esté avancez pour achatez du fer et du charbon pour faire les ferreures du segond engin que on fait près le portal Saint Aulbin pour tyre la pierre et groys desdits fossés comme apert par quictance signée desdits Estot, Audouyn et Henry le $\mathrm{XI}^{\mathrm{e}}$ jour dudit moais de juillet l'an dessusdit mil IIII ${ }^{\mathrm{cc}}$ LXXVIII cy rendue pour ce IX l. tournois."

127. Ibid., $\mathrm{f}^{\circ} 49 \mathrm{v}^{\circ}$.

128. Ibid., $\mathrm{f}^{\circ} 75$.

129. Ibid., $\mathrm{f}^{\circ} 50$. 
dannes ${ }^{130}$ ", ces outils servant à creuser ou à tailler les pierres, dont il est précisé qu'elles peuvent être " larges " ${ }^{131}$. On imagine assez bien ces chantiers où de toutes parts, les ouvriers s'affairent à pointer, creuser, visser et marteler.

\section{Des outils pour transporter et porter}

Lucas Goret touche trente-cinq sous tournois pour " huyt cyvieres fonces et sept cyvieres à bastons ${ }^{132}$ ", Guionne femme d'Yvonnet Letart gagne quatre deniers tournois pour "seix cyvieres à braz et deux à rouelles ${ }^{133}$ "; les ouvriers ont en effet besoin de matériel pour transporter sur le chantier les pierres et le mortier. Si certains portent à bout de bras ces charges lourdes, d'autres s'équipent de " hottes " à porter sur le dos : Jean de Bourc gagne ainsi onze sous cinq deniers tournois pour " pour cinq hotes qu'il a baillé pour les ouvraiges desdits fosse ${ }^{134}$ " tandis que Jean du Rosay, explicitement denommé "faiseur de hotes " est rémunéré quarante-six sous tournois pour les "XIII hotes garnies de bretelles ${ }^{135}$ ” qu'il a fournies.

Un article du compte mentionne également l'acquisition pour quarante sous tournois de " deux grans cuviers et quatre petiz à mecptre l'eau pour estaindre la chaux ${ }^{136}$ ". La mise en œuvre de tels travaux est visiblement coûteuse en main-d'œuvre, en matières premières et en outillage. Par exemple, l'acquisition d'un bédane, cet outil servant à extraire la pierre, coûte quatre sous deux deniers, ce qui équivaut au salaire de deux jours de travail.

130. Ibid., $\mathrm{f}^{\circ} 32$. Le bec-d’âne ou bédane est un burin ou ciseau.

131. Ibid., $\mathrm{f}^{\circ} 49 \mathrm{v}^{\circ}$ : " Audit Gervaise Vrigne a esté paié par ledit Perier commissaire dessusdit la somme de cent onze soulz huyt deniers tournois pour doze becdannes à v soulz la piece, pour xx pointes aguysés à I denier la piece, pour ung gros marteau à cassez les pierres XX soulz, pour III $^{\mathrm{cc}}$ chevilles de fer pour coustre les carreaulx et lyens du grant pont du portal Saint Aulbin et d'un des ponts du portal Saint Michel xxx soulz tournois comme apert par quictance signée desdits Gillebert et Audouyn et de Jehan Nicholas le penultième jour dudit moais de may l'an dessusdit mil IIII ${ }^{\mathrm{cc}}$ LXXVIII cy rendu pour ce CXI s. VIII d. tournois " et $\mathrm{f}^{\circ} 54$ : "À Gervaise Vrigne a esté paié par ledit Perier commissaire dessusdit par le commandement et ordennance de mondit sieur le maire la somme de neusf livres quinze soulz oboles pour sept vigns neusf livres et demie de fer oupvré qu'il a baillé pour ledit engin du portal Saint Aubin qui estoit rompu au pris de neusf deniers tournois chacune livre pour doze picquetz pour les perriers au pris de cinq soulz tournois la piece pour une lieue de feu ouvré pesant vignt et cinq livres au pris de sept deniers oboles la livre pour lesdits perriers pour deux becdannes larges pour lesdits perriers qu'il a acerez pour chacun becdanne xx deniers pour quarante et quatre pointes de picquetz qu'il a agusés par lesdits perriers chacune pointe I denier comme apert par quictance signée desdits Gillebert, Audouyn, Thenin et Nicholas ledit $\mathrm{VI}^{\mathrm{e}}$ jour de juign l'an dessusdit mil IIII ${ }^{\mathrm{cc}}$ LXXVIII cy rendue pour ce IX $\mathrm{l}$. XV s. oboles tournois. "

132. Ibidem, $\mathrm{f}^{\circ} 31$.

133. Ibid., $\mathrm{f}^{\circ} 51$.

134. Ibid., $\mathrm{f}^{\circ} 31 \mathrm{v}^{\circ}$.

135. Ibid., $\mathrm{f}^{\circ} 32$.

136. Ibid., $\mathrm{f}^{\circ} 62 \mathrm{v}^{\circ}$. 


\section{Le coût du chantier}

Les travaux coûtent cher à plusieurs égards. En plus des salaires et des dépenses liées à l'achat de matériaux qui représentent l'essentiel de la somme déboursée par la mairie, il faut encore verser à Jean Perier pour " ses gaiges, peines et sallaires d'avoir fait la recepte et paiemens de ce present compte recuilly ses aquitz sur ce necessaires par ordonnance de messieurs de la maerie ${ }^{137}$ " douze livres dix sous tournois tandis que " pour la minute ${ }^{138}$, grosse ${ }^{139}$, facon et reddicion de ce present compte et du double d'iceluy ${ }^{140}$ ", ce sont cent sept sous six deniers qui doivent être acquités.

La balance globale des comptes se présente de la manière suivante : les recettes s'élèvent à mille six cent quarante et une livres trois sous neuf deniers ${ }^{141}$ tandis que les dépenses se montent à mille six cent soixante et onze livres trois sous deux deniers, ce qui équivaut à environ cent quarante salaires de base, celui d'un besson par exemple, pendant six mois ${ }^{142}$. En l'état, la mairie avait donc encore une dette à honorer envers Jean Perier de vingt-neuf livres dix-neuf sous dix deniers ${ }^{143}$. Comme l'atteste l'article suivant extrait des délibérations du corps de ville datées d'octobre 1493, la mairie est toujours débitrice de cette somme envers Perier :

\section{Ibid., f ${ }^{\circ} 100$.}

138. La minute renvoie à l'original d'un acte authentique.

139. La grosse est la copie certifiée conforme revêtue de la formule exécutoire.

140. Bib. mun. d'Angers, ms 1148 (946), $\mathrm{f}^{\circ} 100$.

141. Gervaise Lecamus, échevin, verse 1355 livres; le maire d'Angers et ledit Lecamus versent ensuite 240 livres 3 sous 9 deniers puis Jehan de la Rivière échevin apporte à son tour 46 livres.

142. À titre d'exemple, à Tours, la construction de deux tours, dans les années 1470 a coûté 3794 livres : les salaires représentent $53 \%$ de la somme totale, le reste étant dévolu à l'achat des matières premières. Les auteurs précisent que " le total des recettes de la ville pour les années 1470-1473 s'élevant à 21457 livres 5 sous 1 denier, la construction des deux tours représente donc environ $17,5 \%$ du total des recettes pour ces quatre années " (RodiER, Xavier et THomas, Frédéric, " La Tour de la rue Chièvre, sur le terrain et dans les comptes de la ville ", dans GaLINIÉ, Henri (dir.), Tours antique et médiéval. Lieux de vie. Temps de la ville. 40 ans d'archéologie urbaine, numéro spécial de la collection "Recherches sur Tours ", Tours, 2007, p. 304-307). Dans le même ordre d'idées, à Nantes, la construction de la porte Saint-Pierre, qui s'étend de 1479 à 1483, est estimée à 6500 livres (ARMIDE, Aurélien, La porte Saint Pierre à Nantes..., op. cit., p. 41).

143. À cette époque, il est assez courant de voir des individus (marchands, bourgeois, échevins...) avancer sur leurs fonds propres de l'argent remboursé plus tard, comme c'est ici le cas, par le corps de ville. Il s'agit là d'un aspect classique du fonctionnement des finances municipales, ce dont rendent d'ailleurs très bien compte les registres de délibération du conseil. Par exemple, Jean Fallet, prévôt, propose à plusieurs reprises entre 1484 et 1487 des avances de fonds au corps de ville contre promesse de remboursement (Arch. mun. d'Angers, BB2 : $f^{\circ} 52, f^{\circ} 53, f^{\circ} 54 v^{\circ}, f^{\circ} 57, f^{\circ} 58, f^{\circ} 60 \mathrm{v}^{\circ}, f^{\circ} 62, f^{\circ} 64$, $\mathrm{f}^{\circ} 69, \mathrm{f}^{\circ} 75 \mathrm{v}^{\circ}, \mathrm{f}^{\circ} 77 \mathrm{v}^{\circ}, \mathrm{f}^{\circ} 86, \mathrm{f}^{\circ} 94 ; \mathrm{BB} 4, \mathrm{f}^{\circ} 17 \mathrm{v}^{\circ}, \mathrm{f}^{\circ} 20, \mathrm{f}^{\circ} 67$ et BB5, $\left.\mathrm{f}^{\circ} 52\right)$, tandis que l'on découvre dans les années 1489-1490 que Jean Lepaige et Pierre Thévin ont fait quant à eux une avance de 500 écus au corps de ville en 1473 (Arch. mun. d'Angers, BB7, f ${ }^{\circ} 29 \mathrm{v}^{\circ}$ et $\mathrm{f}^{\circ}$ 42). Il est également à noter des cas de dons totalement gratuits consentis au corps de ville. Ainsi, Gervaise Lecamus, qui apparaît dans le compte de Jean Perier, fait installer, en 1487, dans sa rue, sur ses deniers personnels, une chaîne pour la sécurité nocturne (Arch. mun. d'Angers, BB4, f ${ }^{\circ} 75$ ). 
"Par Jehan Perier, marchant, demeurant en ceste ville d'Angers, a esté presenté requete avecques noz lectres d'ordonnance d'autreffois atachés à icelle par laquelle requete il disoit que, dès le XXVII jour de mars mil cccc LXXVIII, il avoit esté commis à faire le poyement des oupvraiges qui furent faiz lors es foussez et douves du portal Saint Aulbin des deniers que seroient mis en ses mains. Desquelx ouvraiges, il rendit compte et par l'arrest d'iceluy avoit esté trouvé luy estre deu pour plus avoir mis que receu la somme de XXIX livres XIX sols X deniers oboles, laquelle fut mandé au receveur des deniers de la ville luy paier ce que ledit receveur n'avoit depuis peu faire obstant plusieurs charges que depuis estoient sourvenues à ladite ville et par ce, requeroit avoir assignacion et paiement d'icelle. Laquelle requete, veue ensemble nosdites lectres d'ordonnance atachés à icelle pour ces causes, a esté et est derechef appointé et ordonné que ledit Perier sera payé de ladite somme de XXIX livres XIX sols X deniers oboles sur et des deniers de la Cloaison d'Angers de ceste presente année et sur le tiers quartin d'icelle ou plus tost si faire ce peut; commensant ladite année le premier jour de ce moys dernier passé et sans de ceste presente assignacion luy faire aucune rompture et partant etc. ${ }^{144}$."

Si l'on en croit ce document, tout est mis en œuvre pour que Jean Perier touche au plus vite ses gages mais il n'y a aucune trace postérieure de ce paiement.

\section{Quelques aspects du quotidien d'un chantier à la fin du Moyen Âge}

Au-delà de l'aspect très technique de cette source documentaire, le compte de Jean Perier permet d'appréhender des éléments relatifs au quotidien des individus qui ont fréquenté ce chantier. Tour à tour, il est ainsi possible d'entrevoir l'atmosphère régnant sur les chantiers, de découvrir que cet univers n'est pas totalement masculin et d'appréhender les risques auxquels s'exposent les travailleurs.

\section{Un chantier sous surveillance : bagarres, désertions et tensions}

Un passage du compte laisse entendre que l'entente et la cordialité entre les ouvriers travaillant à la remise en état des fossés et des murs d'enceinte ne sont pas toujours de mise:

" Jehan de France a esté paié par ledit Perier commissaire dessusdit la somme de quarante et cinq soulz tournois pour seix chesgnes de fer du commandement et ordennance de mondit sieur le maire lesquelles chesgnes sont ordennées pour mecptre les ouvriers qui s'entre batent et celx qui faillent de leurs journés comme apert par quictance du $\mathrm{x}^{\mathrm{e}}$ jour dudit moais de may l'an dessusdit mil IIII ${ }^{\mathrm{cc}}$ LXXVIII signée dudit Estot et Nicholas et de Jehan Henry cy rendue pour ce XLV s. tournois ${ }^{145}$."

Les autorités prennent visiblement des dispositions pour gérer au mieux les éventuels problèmes qui pourraient se présenter à elles : punir

144. Arch. mun. d'Angers, BB 8, octobre 1493.

145. Bib. mun. d'Angers, ms 1148 (946), f $43 \mathrm{v}^{\circ}$. 
les bagarres et dissuader les éventuelles velléités de désertion. Le maintien de l'ordre est une donnée qui semble prise en compte par les commanditaires des travaux. Cela révèle que les travailleurs sont surveillés même si l'on doit toutefois signaler qu'à aucun moment, il n'est fait état de l'utilisation de ces moyens pour le moins coercitifs contre tel ou tel individu.

\section{Des femmes dans un univers d'hommes}

Bien qu'étant en grande minorité par rapport à leurs homologues masculins, des femmes, une dizaine au total, émergent de la comptabilité de Jean Perier. Au même titre que les hommes, certaines d'entre elles assurent l'approvisionnement des chantiers en matériaux. Par exemple, Guionne, la femme d'Yvonnet Letart, reçoit, le 16 mai, onze sous pour " trois cyvieres roulleresses ${ }^{146}$ ", le 30 mai vingt-deux sous quatre deniers " pour seix cymieres à braz et deux à rouelles ${ }^{147}$ " et le 20 juin quinze sous " pour quatre cyvieres roulereses qu'elle a baillées pour l'ouvrage desdits fosés ${ }^{148}$ ", tandis que Coline, la femme de Jean de Bongne, Beatrisse, la femme de Guillaume Beze et Ysabeau la Regnaude perçoivent la somme de quarante-huit sous tournois " pour huyt tumbelerés de chaulx estainte ${ }^{149}$ ".

Elles peuvent également prendre en charge le service de l'eau, à l'instar de Jeanne la Rogere qui approvisionne en eau durant plusieurs semaines les "gens besongnans esdits fossez " et gagne pour cela plus de cent sous ${ }^{150}$. Ysabeau la Cerisiere touche quant à elle neuf sous six deniers " pour cinquante et sept pintes de vin que elle a bailé aux ouvriers desdits fosez ${ }^{151}$ ". Le service de l'eau n'est toutefois pas une tâche qui incombe uniquement aux femmes puisque le compte mentionne également la présence de Nonner Guyeur, un homme, qui sert aussi l'eau aux ouvriers pendant une semaine et gagne un salaire équivalent à celui versé aux femmes, soit cinq sous ${ }^{152}$.

Les femmes sont aussi très présentes lorsqu'il s'agit de loger, nourrir, blanchir les condamnés au travail forcé. Perrine la Girarde est ainsi payée sept sous six deniers le 18 juillet " pour avoir blanchy et relavé le linge de l'aumosnerie de Brechigne ou couschent XxIIII compaignons qui besongnent esdits fosez ${ }^{153}$ " et de nouveau sept sous six deniers le premier août " pour avoir logé XXIIII compaignons qui besongnent esdits fosez ceste sepmaine et leur a baillé lytz et draps ${ }^{154}$ ", tandis qu'Ysabeau la Cerisiere gagne soixantetrois sous six deniers " pour la despence de vingnt et quatre hommes qui

146. Ibidem, $\mathrm{f}^{\circ} 45$.

147. Ibid., $\mathrm{f}^{\circ} 51$.

148. Ibid., $\mathrm{f}^{\circ} 61 \mathrm{v}^{\circ}$.

149. Ibid., $\mathrm{f}^{\circ} 50 \mathrm{v}^{\circ}$.

150. Ibid., $\mathrm{f}^{\circ} 51 \mathrm{v}^{\circ}, \mathrm{f}^{\circ} 53 \mathrm{v}^{\circ}, \mathrm{f}^{\circ} 57 \mathrm{v}^{\circ}, \mathrm{f}^{\circ} 60 \mathrm{v}^{\circ}, \mathrm{f}^{\circ} 64, \mathrm{f}^{\circ} 66, \mathrm{f}^{\circ} 72 \mathrm{v}^{\circ}, \mathrm{f}^{\circ} 75 \mathrm{v}^{\circ}, \mathrm{f}^{\circ} 78 \mathrm{v}^{\circ}, \mathrm{f}^{\circ} 82$, $\mathrm{f}^{\circ} 84 \mathrm{v}^{\circ}, \mathrm{f}^{\circ} 88, \mathrm{f}^{\circ} 91 \mathrm{v}^{\circ}, \mathrm{f}^{\circ} 95, \mathrm{f}^{\circ} 97$ et $\mathrm{f}^{\circ} 98$.

151. Ibid., $\mathrm{f}^{\circ} 69$.

152. Ibid., $\mathrm{f}^{\circ} 30 \mathrm{v}^{\circ}$.

153. Ibid., $\mathrm{f}^{\circ} 75$.

154. Ibid., $\mathrm{f}^{\circ} 82 \mathrm{v}^{\circ}$. 
ont esté mis à besongner esdits fosez par justice lesquelx estoient oyseulx et ont besongné ceste sepmaine chacun par cinq jours et lesquelx sont aux despens sur les deniers de la ville ${ }^{155} "$.

Il reste une dernière tâche qui incombe aux femmes, celle de prendre soin des ouvriers blessés; elles remplissent alors le rôle de panseuse. Jeanne Boterelle gagne ainsi dix sous en juin " pour avoir pencé par doze jours Colas Voysin qui fut blecié à l'engin ${ }^{156}$ " et vingt sous en juillet " pour avoir pencé ledit Marcade qui fut blecié à l'engin par le temps d'un mois escheu mercredi derrain ${ }^{157}$ ". Ces mentions sont rares dans la documentation angevine. Nous ne connaissions, jusqu'à présent, qu'une barbière mentionnée à la fin du XIII ${ }^{\mathrm{e}}$ siècle ${ }^{158}$.

\section{"Les risques du métier " : la prise en charge des blessés}

Chutes mortelles, jambes et bras cassés, corps malmenés sous les roues des charrettes ou après l'effondrement d'un pan de mur mal étayé, brûlures à la chaux, enlisements dans les fossés, les risques et les accidents liés au travail sur les chantiers sont facilement imaginables. L'état de santé des ouvriers transparaît dans le compte, à l'instar de Jean Bernier qui, " pour une journée qu'il a servy les maczons et s'en alla malade ${ }^{159}$ " a été payé le 27 juillet deux sous tournois. Mathurin Bregeot, boucher de son état, gagne quant à lui dix-huit sous quatre deniers " pour deux moutons qui furent prins de luy pour avoir les peaulx pour mecptre sur l'un des deux hommes qui fut blecié à l'engin des douves du portal Saint Aulbin dont la char fut donnée aux barbiers et aux femmes qui pencent lesdits bleciez ${ }^{160}$ "; Jehan Plumneul et Pierre Arthus, barbiers, sont rémunérés conjointement à quatre reprises entre le 13 juin et le 4 juillet pour les bons soins qu'ils ont, eux aussi, prodigués à Jean Marcade et Colas Voysin blessés au cours du chantier de remise en état des fossés de l'enceinte angevine ${ }^{161}$. La présence

155. Ibid., $\mathrm{f}^{\circ} 69 \mathrm{v}^{\circ}$.

156. Ibid., $\mathrm{f}^{\circ} 52 \mathrm{v}^{\circ}$.

157. Ibid., $\mathrm{f}^{\circ} 75$.

158. WicKENSHEIMER, Ernest, Dictionnaire biographique des médecins en France au Moyen Âge, Genève, Droz, 1979, t. 2, p. 750.

159. Ibid., $\mathrm{f}^{\circ} 82$.

160. Ibid., $\mathrm{f}^{\circ} 48 \mathrm{v}^{\circ}$.

161. Ibid., $\mathrm{f}^{\circ} 57 \mathrm{v}^{\circ}$ : " À Jehan Plumneul et Pierre Arthus barbiers a esté payé par ledit Perier commissaire dessusdit la somme de quatre livres quinze soulz tournois par le commandement et ordennance de mondit sieur le maire pour avoir pencé Marcade et Colas Voysin qui furent bleciez à l'engin ung moys à ou environ et ont fourny de linge et autres choses necessaires comme appert par quictance signée desdits Estot, Thenin et Audouyn le XIII ${ }^{\mathrm{e}}$ jour dudit moais de juign l'an dessusdit mil IIII ${ }^{\mathrm{cc}}$ LXXVIII cy rendue par ce IIIIL. XV s. tournois "; $\mathrm{f}^{\circ} 61$ : " À Pierre Arthus et Jehan Plummeul barbiers par le commandement et ordennance de mondit sieur le maire la somme de vignt soulz tournois laquelle somme a esté paiée par ledit Perier commissaire dessusdit ausdits barbiers pour avoir pencé Jehan Marcade qui fut blecié à l'engin comme apert par quictance signée desdits Gillebert, Audouyn et Nicholas le sabmedi xx jour dudit moais de juign l'an dessusdit mil ${ }_{\text {IIII }}{ }^{\text {cc }}$ LXXVIII cy rendue pour ce XX s. tournois "; $\mathrm{f}^{\circ} 63$ : " $\grave{A}$ Jehan Plumneul et Pierre Arthus 
de ces deux barbiers n'a du reste rien de surprenant. En effet, si les médecins observent les corps et les comportements et dressent les diagnostics médicaux, on attend des barbiers qu'ils prodiguent les premiers soins aux blessés, les assistent à l'occasion dans leur convalescence et pratiquent dans certains cas, eu égard à leur dextérité à manier les instruments tranchants, les différentes ouvertures pour rendre possible l'observation précise des organes et autres viscères.

Comme l'atteste le compte de Jean Perier, les deux blessés du chantier ont été pris en charge par différentes personnes durant plusieurs semaines, ce qui est le signe d'un accident sans doute grave. Ainsi, Jeanne Boterelle touche dix sous " pour avoir pencé par doze jours Colas Voysin qui fut blecié à l'engin ${ }^{162}$ ". Le second blessé est, quant à lui, soigné pendant quinze jours par Jeanne Marée, soins pour lesquels elle est rémunérée quinze sous. Mais les femmes ne sont pas les seules à prendre en charge les blessés comme le montrent les quatre paiements qui ont été versés entre le vingt-trois mai et le dix-huit juillet à Bastien Vayre, qui a également hébergé Jean Marcade et Colas Voysin ${ }^{163}$. La prise en charge des deux blessés a

barbiers par le commandement et ordennance de mondit sieur le maire a esté paié par ledit Perier commissaire dessusdit la somme de vignt soulz tournois pour avoir pencé ceste sepmaine Jehan Marcade qui fut blecié audit engin du portal Saint Aubin comme apert par quictance signée desdits Gilebert, Audouyn et Nicholas le XXVII jour dudit moais de juign l'an dessusdit mil IIII ${ }^{\mathrm{cc}}$ LXXVIII cy rendue pour ce XX s. tournois "; $\mathrm{f}^{\circ} 68$ : " À Jehan Plumneul et Pierre Arthus barbiers par le commandement et ordennance de mondit sieur le maire a esté payé par ledit Perier commissaire dessusdit la somme de vignt soulz tournois pour avoir pencé ceste sepmaine Jehan Marcade qui fut blecié audit engin du portal Saint Aubin comme apert par quictance signée desdits Estot, Thenin et Audouyn ledit IIII ${ }^{\mathrm{e}}$ jour dudit moais de juillet l'an dessusdit mil IIII ${ }^{\mathrm{cc}}$ LXXVIII cy rendue pour ce XX s. tournois ». Quant à Jean Plumneul, il reçoit un paiement supplémentaire, voir $\mathrm{f}^{\circ} 74:$ : À Jehan Plumneul a esté payé par ledit commissaire la somme de quarante soulz tournois pour avoir pencé Marcade qui fut blecié à l'engin par deux sepmaines comme apert par quictance signée desdits Gillebert, Fleuri et Nicholas le XVIII ${ }^{\mathrm{e}}$ jour dudit moais de juillet l'an dessusdit mil IIII ${ }^{\mathrm{cc}}$ XXVIII cy rendue par ce XL s. tournois."

162. Ibidem, $\mathrm{f}^{\circ} 52 \mathrm{v}^{\circ}$.

163. Ibid., $\mathrm{f}^{\circ} 47$ : "À Bastien Vere a esté payé par ledit Perier commissaire dessusdit la somme de quarante soulz tournois sur ce que luy peut ou pourra estre deu pour la despence de Colas Voysine et Marcade qui furent bleciez à l'engin comme apert par quictance signée desdits Gillebert, Audouyn et Fleuri le XxIII ${ }^{\mathrm{e}}$ jour dudit moais de may

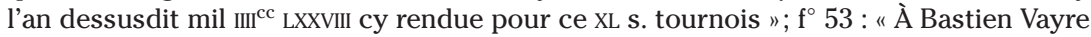
demorant à l'oustelerie de l'ymage Saint Maurice près le portal Saint Aulbin a esté paié par ledit Perier commissaire dessusdit la somme de quatre livres quatre soulz ung denier tournois oultre quarante soulz que ledit Vayre a euz dudit Perier paravant ce jour dont il a baillé quictance audit Perier lesquelles sommes mondit sieur le maire luy avoit ordenné pour la despence de Jehan Marcade et Colas Voysine qui furent bleciez à l'engin dudit portal Saint Aulbin; et pour la despence de Jehanne Macée et Jehanne Boterelle qui ont pencé lesdits bleciez depuis le jour qu'ilz furent bleciez jucques aujourd'uy qui sont XVI jours et aussy pour les avoir logez en sa maison comme apert par quictance signée desdiz Gillebert, Thenin et Nicholas le III ${ }^{\mathrm{e}}$ jour dudit moais de juign l'an dessusdit mil IIII ${ }^{\mathrm{cc}}$ LXXVIII cy rendue pour ce IIII l. IIII s. I d. tournois "; $\mathrm{f}^{\circ} 56 \mathrm{v}^{\circ}$ : " À Bastien Vere par le commandement et ordennance de mondit sieur le maire a esté payé par ledit Perier commissaire dessusdit la somme de trente soulz tournois sur ce que luy peut ou pourra estre deu pour la despence 
également nécessité, au moins pour l'un d'entre eux, des médicaments; Guillaume Janvier est ainsi payé au mois de juillet six sous dix deniers " pour apotiquererie qu'il a baillé pour Marcade qui fut blecié à l'engin ${ }^{164}$ ". Si le travail est harassant et parfois dangereux sur le chantier, il reste que le registre atteste d'un souci certain pour les ouvriers qui, des heures durant, travaillent à la restauration des fossés et des murs de l'enceinte angevine; les autorités dépensent plus de vingt-six livres en soins médicaux et prise en charge de la convalescence des blessés ${ }^{165}$.

Jean Perier livre là un bien beau document retraçant la vie quotidienne d'un chantier dans tous ses aspects techniques, économiques et humains. Il permet d'appréhender le travail des ouvriers effectué sans doute dans des conditions dures et sous le commandement d'hommes compétents et chevronnés. Il donne à voir toute l'infrastructure financière qu'il a fallu mettre en place afin de mener à bien ces travaux d'une ampleur, au demeurant, non négligeable : nous avons pu mesurer les dépenses effectuées de l'ordre de plusieurs centaines de livres et avons découvert la grande qualité technique de la comptabilité de Jean Perier, lequel donne l'impression d'avoir apporté un soin tout à fait particulier à son entreprise comptable.

Au Moyen Âge, l'entretien des fortifications constituait un poste de dépense important pour de nombreuses villes. Cette source, replacée dans le contexte des comptabilités municipales, illustre pleinement la hauteur de l'investissement consenti par les villes pour assurer leur sécurité, dès lors que les menaces devenaient tangibles. Il fallait sans tarder remettre en état les parties dégradées par le temps et le manque d'entretien. Il fallait aussi adapter le système défensif aux progrès de l'artillerie à poudre, alors en pleine évolution, et dont la puissance de feu devenait redoutable en cas de

de Marcade qui fut blecié audit engin de la femme dudit Marcade et de Jehanne Boterelle qui sont ordennées par mesdits sieurs pour le pencer comme apert par quictance signée desdits Gillebert, Thenin et Audouyn le XIII ${ }^{\mathrm{e}}$ jour dudit moais de juign l'an dessusdit mil IIII ${ }^{\mathrm{cc}}$ LXXVIII cy rendue par ce XXX s. tournois " et $\mathrm{f}^{\circ} 74 \mathrm{v}^{\circ}$ : " À Bastien Vere par le commandement et ordennance de mondit sieur le maire par ledit Perier commissaire dessusdit la somme de seix livres quinze soulz tournois pour la despence de Marcade qui fut blecié à l'engin et de celx qui ont est à le pencer et garder de toute laquelle despence ledit Vere c'est tenu content et bien paié omme apert par quictance signée desdits Estot, Audouyn et Fleuri le sabmadi XVIII jour dudit moais de juillet l'an dessusdit mil IIII $^{\mathrm{Cc}}{ }^{\mathrm{CXXXVIII}}$ cy rendue pour ce VI l. XV s. tournois".

164. Ibidem, $\mathrm{f}^{\circ} 72 \mathrm{v}^{\circ}$.

165. En 1521 encore, la mairie d'Angers octroie une aide à un homme de condition modeste qui s'est gravement blessé alors qu'il travaillait au curage des fossés de la ville : "Pour ce qu'il a esté rapporté par mondit sieur le maire que ung paouvre homme impotent nommé Allain Cirart quy a par cy davant servy la ville au curement et nectoyement des rues d'icelle et audit service dit y avoir perdu une jambe, a esté [?] a requis ledit Cirart à mesdits sieurs que leur plaisir fust luy donner et aulmosner une robe. Ouye laquelle requete, a esté ordonné estre baillé par le receveur des deniers communs de ladite ville audit Cirart jusques à deux aulnes de bureau pour faire une robe du pris de douze solz six deniers tournois " (Arch. mun. d'Angers, BB 17, f ${ }^{\circ}$ 141, délibérations du mardi premier octobre 1521). 
siège $^{166}$. Les sommes englouties, à Angers comme dans toutes les grandes villes, ont contraint les municipalités à adopter des comptabilités de plus en plus élaborées afin de suivre pas à pas les dépenses et de s'assurer de la qualité des travaux réalisés ${ }^{167}$. La dépense de l'argent public fait visiblement l'objet d'une surveillance étroite.

Par ailleurs, ce chantier, comme beaucoup d'autres à cette époque, réunit des individus aux origines sociales (des édiles aux marginaux) et géographiques (Angers et son arrière-pays) diverses. Le nombre des intervenants, ramené à la population de la ville, illustre l'impact de tels chantiers qui devaient rythmer une partie de la vie de la cité et fournir une quantité de travail considérable, dynamisant l'activité économique locale par les nombreux salaires versés. Si deux blessés sont à déplorer, les travaux semblent être allés dans l'ensemble assez vite et s'être déroulés sans encombre : le compte ne dit rien d'éventuels problèmes dans la conduite et la mise en œuvre des opérations. Jean Perier a, semble-t-il, su superviser d'une main de maître cet épisode de remise en état des fossés et du mur d'enceinte de la ville d'Angers.

166. À cet égard, voir le travail de Crouy-ChANEl (de), Emmanuel, Le canon jusqu'au milieu du XVI siècle (France, Bretagne et Pays-Bas bourguignons), thèse d'histoire, université Paris I, 2014, et celui de MARTINEAU, Jocelyn, "Les tours à canon du duché de Bretagne au XV ${ }^{\mathrm{e}}$ siècle ", dans Artillerie et fortifications 1200-1600, Rennes, PUR, 2011, p. 191-214. 167. Le travail de Jean-Pierre LEGUAY rend très bien compte de cela, voir La ville de Rennes au XV siècle à travers les comptes des miseurs, Paris, C. Klincksieck, 1969. 


\section{RÉSUMÉ}

En 1478, Jean Perier est missionné par le corps de ville d'Angers pour engager la remise en état d'une partie des fossés et des murs de l'enceinte urbaine. Sa comptabilité, un registre d'une centaine de folios, fait état de nombreuses informations concernant l'organisation matérielle du chantier, l'acheminement des matériaux nécessaires à la réalisation des travaux ou bien encore les corps de métier engagés. Au fil des pages, l'auteur de ce compte nous fait entrer de plein pied dans cet univers de travail où se côtoient des individus issus d'horizons socio-économiques différents. À cet égard, il faut mentionner la présence de plusieurs hommes condamnés par la justice à venir effectuer des périodes de travail, s'étalant parfois sur toute une semaine, dimanche compris. Jean Perier à qui revient la charge de suivre le chantier et de payer les travaux au fur et à mesure de leur réalisation se fait également le porte-parole d'un quotidien fait de temps à autre d'accidents et de blessures. Ces quelques pages font également une place aux femmes, présentes pour distribuer l'eau ou prendre en charge les blessés.

\section{ABSTRACT}

In 1478, Jean Perier was appointed by the council of the city of Angers to begin the rehabilitation of a part of the ditches and walls within its boundaries. His accounts, a register of over a hundred folios, reported valuable information regarding the material organisation of the construction site, the delivery of the materials required in order to carry out the work, as well as the construction trades involved in the project. Throughout the register, the author of this account allows us to enter fully into the universe of this restoration project for which individuals from different socioeconomic backgrounds worked side by side. In this regard, the presence of several men sentenced by the court to undertake periods of work, sometimes stretching over an entire week, including Sundays, must also be mentioned. Jean Perier, who was in charge of monitoring the construction site and paying for work as it progressed, also became the spokesman for the everyday life of the construction site, sometimes including accidents and injuries. These pages also mention the women whose role was to distribute water or to care for the wounded. 